\title{
THE STRUTINSKY METHOD AND ITS FOUNDATION FROM THE HARTREE-FOCK-BOGOLIUBOV APPROXIMATION AT FINITE TEMPERATURE
}

\author{
M. BRACK \\ Institut für Theoretische Physik, Universität Regensburg, D-8400 Regensburg, W.-Germany \\ and \\ P. QUENTIN \\ Institut Max von Laue-Paul Langevin, BP 156X, F-38042 Grenoble, France
}

Received 29 July 1980

\begin{abstract}
Strutinsky's shell-correction method is investigated in the framework of the microscopical Hartree-Fock-Bogoliubov method at finite temperature (HFBT). Applying the Strutinsky energy averaging consistently to the normal and abnormal density matrices and to the entropy, we define a self-consistently averaged HFBT system as the solution of a variational problem. From the latter we derive the generalized Strutinsky energy theorem and the explicit expressions for the shell correction of a statistically excited system of BCS quasiparticles. Using numerical results of HF calculations, we demonstrate the convergence of the Strutinsky expansion and estimate the validity of the practical shell-correction approach. We also discuss the close connections of the Strutinsky energy averaging with semiclassical expansions and their usefulness for solving the average nuclear self-consistency problem. In particular we argue that the Hohenberg-Kohn theorem should hold for the averaged HFBT system and we thus provide a justification of the use of semiclassical density functionals.
\end{abstract}

\section{Introduction}

Among the many contributions of Sven Gösta Nilsson to nuclear physics, one may single out a long-standing effort to determine microscopically deformation energy surfaces. Taking full advantage of his simple yet fruitful ansatz for the mean field of a deformed nucleus ${ }^{1}$ ) he has together with Mottelson attempted to give an account of the wealth of experimental data on permanent nuclear deformations in the late fifties ${ }^{2}$ ). The limitations of the approach for deformations as large as those occuring near the fission barrier had soon become evident ${ }^{3-5}$ ). The correct renormalization of the bulk of the deformation energy to reproduce the empirically known liquid drop behaviour has been later proposed by Strutinsky ${ }^{6-7}$ ). Among other groups calculating at the same time fission barriers for actinide and superheavy nuclei ${ }^{8-12}$ ), the Lund-Warsaw group ${ }^{13}$ ) has been the first (in 1969) to present an extensive account of deformation energy curves using the Strutinsky method. 
Further improvements, consisting in the inclusion of left-right reflexion ${ }^{14}$ ) and axial ${ }^{15}$ ) asymmetries have followed shortly after in their work. In the last five years, the same method has been applied for computing deformation energy surfaces at finite angular momenta ${ }^{16}$ ).

In view of the considerable effort devoted by Sven Gösta Nilsson and his group, as well as by many others, to the difficult task of microscopically determining potential energy surfaces within the Strutinsky method, it is of particular importance to investigate in great details the validity of the whole approach. This has already been studied within the Hartree-Fock approximation by various authors ${ }^{17-21}$ ).

It has appeared increasingly clear that it is necessary to properly introduce pairing correlations ${ }^{22-24}$ ) in the calculation of potential energy surfaces. Only one attempt has been made so $\mathrm{far}^{25}$ ) to formulate the Strutinsky method consistently within the Hartree-Fock-Bogoliubov (HFB) framework ${ }^{24}$ ). Usually, the pairing effects were included in the shell-correction method in the phenomenological BCS approximation with a constant pairing matrix element $G$ [refs. $\left.\left.{ }^{6-8,26}\right)\right]$. It is one of the main purposes of this paper to pursue the studies initiated in refs. ${ }^{25,26}$ ) and to give a fully self-consistent HFB formulation of the Strutinsky method.

In recent years, statistically excited nuclei have been studied within non-selfconsistent ${ }^{26-30}$ ) and self-consistent ${ }^{31-33}$ ) independent-particle approaches. On the other hand a close connexion between the Strutinsky energy smoothing and the finite temperature average was recognized long ago ${ }^{34}$ ) and further studied by various authors ${ }^{25,35-37}$ ). This provides a motivation to study, as we will do here, the Strutinsky energy theorem and energy averaging in the framework of the HFB approximation at finite temperature (HFBT). It is quite obvious that complete HFBT calculations are somewhat heavy to handle and their approximation according to the Strutinsky method is rather welcome. It is thus necessary to discuss in some detail the many assumptions inherent to such calculations. Among them the most promising might be those taking full advantage of the basic relation between the Strutinsky method and the semiclassical approximation ${ }^{7-8,38-43}$ ), once some remaining deficiencies of current effective interaction parametrizations [see e.g. ref. ${ }^{44}$ )] have been removed.

Our paper is organized as follows. In sect. 2 we summarize the most important definitions and assumptions made in the HFB approach and its extension to finite temperatures (HFBT), mainly in order to fix the nomenclature for the following treatment. An extensive sect. 3 is devoted to the self-consistent incorporation of the idea of Strutinsky averaging into the HFBT framework. As a result of these investigations, we shall derive the so-called Strutinsky energy theorem, which gives the formal basis of the shell-correction approach, for the general case of an excited system of quasiparticles. A more detailed guide to sect. 3 is given at its beginning.

The numerical validity of the shell-correction method will be investigated in sect. 4 , based on HF calculations in various situations $(T \neq 0$ and $T=0$, with and without 
pairing). We will demonstrate that the Strutinsky energy theorem is fulfilled to a high numerical accuracy in a self-consistent treatment, and discuss some problems connected to the practical use of phenomenological shell and liquid drop models.

Sect. 5 will finally be devoted to the prospects of using semiclassical methods in solving the (static) nuclear self-consistency problem. Some promising recent developments will be discussed, which aim at the semi-classical determination of selfconsistent average nuclear potentials and deformation energies from a given effective nucleon-nucleon interaction. With that, not only the "ideal" ingredients for a Strutinsky calculation could be given, but also an economical and still selfconsistent alternative to the very time consuming, purely microscopical approach to nuclear deformation energies.

\section{Hartree-Fock-Bogoliubov approximation at finite temperature}

The Hartree-Fock-Bogoliubov wave function results from the minimization of the expectation value of a given effective hamiltonian in an independent quasiparticle state ${ }^{24}$ ). The corresponding quasiparticle operators are defined by the canonical Bogoliubov transformation

$$
\zeta_{i}^{+}=\sum_{\alpha}\left(X_{\alpha i} a_{\alpha}^{+}+Y_{\alpha i} a_{\alpha}\right)
$$

where $a_{\alpha}^{+}, a_{\alpha}$ are single-particle creation and annihilation operators on a given basis. Due to the well-known theorem of Bloch and Messiah ${ }^{45}$ ) one may decompose the transformation (2.1) into the product of three successive transformations:

(i) a unitary transformation from the basis $\{\alpha\}$ to a Hartree-Fock (HF) singleparticle basis $\{i\}$;

(ii) a restricted canonical Bogoliubov transformation [the so-called BogoliubovValatin ${ }^{46,47}$ ) transformation]

$$
\zeta_{i}^{+}=u_{i} a_{i}^{+}-v_{i} a_{i}, \quad \zeta_{i}^{+}=u_{i} a_{i}^{+}+v_{i} a_{i}
$$

where $i$ refers to a state deduced from the state $i$ by time-reversal conjugation (the Bogoliubov-Valatin transformation defines thus BCS quasiparticle states.);

(iii) a unitary transformation among the previously defined quasi-particle states.

The completion of the first two steps corresponds to a self-consistent HF + BCS calculation. From recent extensive numerical HFB calculations ${ }^{48-51}$ ) it turns out that the third part of the Bogoliubov transformation does not bring any substantial changes into the total energy and the normal one-body density. It is therefore justified, as we will mostly do it here, to omit it and restrict the discussion to $\mathrm{HF}+\mathrm{BCS}$ calculations. 
The HFB ground-state wave function $|0\rangle$ corresponds to a vacuum of quasiparticles defined by two one-body densities; a normal one given in any basis by

$$
\rho_{\alpha \beta}=\left\langle 0\left|a_{\beta}^{+} a_{\alpha}\right| 0\right\rangle,
$$

and an abnormal one

$$
\kappa_{\alpha \beta}=\left\langle 0\left|a_{\alpha} a_{\beta}\right| 0\right\rangle .
$$

In terms of these densities one may write the expectation value of the energy (for a hamiltonian including a two-body interaction $\mathscr{V}$ ) as

$$
E_{\mathrm{HFB}}=\langle 0|H| 0\rangle=\operatorname{tr} \mathscr{T} \rho+\frac{1}{2} \operatorname{tr} \operatorname{tr} \rho \overline{\mathscr{V}} \rho+\frac{1}{4} \operatorname{tr} \operatorname{tr} \kappa \overline{\mathscr{V}} \kappa^{*} .
$$

$(\mathscr{T}$ being the kinetic energy operator and $\mathscr{V}$ corresponding to the matrix elements of the antisymmetrized interaction: $\left.\mathscr{V}=\mathscr{V}\left(1+P_{\sigma} P_{\tau} P_{\mathrm{M}}\right)\right)$. Equivalently, upon defining a HF potential $\Gamma$ and a pairing potential $\Delta$ by

$$
\begin{gathered}
\Gamma=\operatorname{tr} \rho \overline{\mathscr{V}}, \\
\Delta=-\frac{1}{2} \operatorname{tr} \kappa \overline{\mathscr{V}}
\end{gathered}
$$

one may write $E$ as

$$
E_{\mathrm{HFB}}=\operatorname{tr}\left(\mathscr{T}+\frac{1}{2} \Gamma\right) \rho-\frac{1}{2} \operatorname{tr} \Delta \kappa^{*}
$$

Note, however, that the definition of $\Gamma$, eq. (2.6), does not hold for a density-dependent interaction $\mathscr{V}(\rho)$ (see the discussion at the end of subsect. 3.5 for this case).

In the restricted $\mathrm{HF}+\mathrm{BCS}$ case, upon further assuming the wave function to be even under time reversal, one may define a real representation of $(\mathscr{T}+\Gamma), \Delta, \rho$ and $\kappa$ such that

$$
\begin{gathered}
(\mathscr{T}+\Gamma)_{i j}=\varepsilon_{i} \delta_{i j} \\
\Delta_{i j}=\delta_{i j} \Delta_{i}=-\frac{1}{2} \sum_{k} u_{k} v_{k}\langle\bar{i}|\overline{\mathscr{V}}| k \bar{k}\rangle \delta_{i j} \\
\rho_{i j}=\delta_{i j} n_{i}=v_{i}^{2} \delta_{i j} \\
\kappa_{i j}=\delta_{i j} k_{i}=u_{i} v_{i} \delta_{T j}
\end{gathered}
$$

where the real numbers $u_{i}$ and $v_{i}$ are those entering the Bogoliubov-Valatin transformation [see eq. (2.2)] and are such that:

$$
\begin{gathered}
u_{\bar{i}}=u_{i}, \quad v_{i}=-v_{i} ; \\
u_{i}^{2}+v_{i}^{2}=1 \\
v_{i}^{2}=\frac{1}{2}\left(1+\frac{\lambda-\varepsilon_{i}}{\mathscr{E}_{i}}\right) .
\end{gathered}
$$


In the equation defining $v_{i}^{2}$, the chemical potential $\lambda$ is determined by imposing that the average number of particles should be equal to the (even) number $N$ of nucleons ${ }^{\dagger}$

$$
\sum_{i} v_{i}^{2}=\operatorname{tr} \rho=N
$$

and $\mathscr{E}_{i}$ is the energy of a one-quasiparticle state,

$$
\mathscr{E}_{i}=\left\langle 0\left|\zeta_{i} H \zeta_{i}^{+}-H\right| 0\right\rangle=\sqrt{\left(\lambda-\varepsilon_{i}\right)^{2}+\Delta_{i}^{2}} \equiv \mathscr{E}\left(\varepsilon_{i}, \lambda, \Delta_{i}\right)
$$

Now we want to deal with excited systems within the independent quasi-particle approximation. If we assume that all the excitation energy is equally shared among all degrees of freedom, the corresponding wave function should be a solution of a canonical thermodynamical equilibrium problem under the constraint that the average number of particles (not quasiparticles!) should be equal to $N$, as proposed by Sano and Yamasaki ${ }^{52}$ ). In other words we would like to minimize the thermodynamical potential

$$
\Omega=\langle H\rangle-T S-\lambda\langle\mathcal{N}\rangle,
$$

where $T$ is the temperature, $S$ the entropy defined ${ }^{\dagger \dagger}$ in terms of the many-body density matrix $\mathscr{D}$ as $-\operatorname{tr}(\mathscr{D} \ln \mathscr{D}), \lambda$ the chemical potential and $\mathscr{N}$ the particle number operator. As is well known [see e.g. ref. $\left.{ }^{22}\right)$ ] the independent quasiparticle approximation consists in replacing $H-\lambda \mathcal{N}$ by

$$
H-\lambda \mathcal{N}=E_{\mathrm{HFB}}+H^{\mathrm{QP}},
$$

with

$$
H^{\mathrm{QP}}=\sum_{i} \mathscr{E}_{i} \zeta_{i}^{+} \zeta_{i}
$$

(The variational definition of the quasiparticle operators $\zeta_{i}, \zeta_{i}^{+}$will be given below.) One is now left with the usual canonical thermodynamical problem for an independent fermion hamiltonian $H^{\mathrm{QP}}$ whose solution $|T\rangle$ is completely defined by its one-body reduced density matrix, diagonal in the quasiparticle basis $\{i\}$ and such that

$$
\left\langle T\left|\zeta_{i}^{+} \zeta_{i}\right| T\right\rangle=n_{i}^{T}=\frac{1}{1+\exp \left(\mathscr{E}_{i} / T\right)} \equiv n^{T}\left(\varepsilon_{i}, \lambda, \Delta_{i}\right)
$$

${ }^{\dagger}$ For simplicity, we assume throughout this article only one kind of nucleons. Sums over $i$ run always explicitly over all single-particle states $|i\rangle$ and their time reversed $\mid i \overrightarrow{\rangle}$.

+† We will skip for simplicity the Boltzmann constant and consequently consider the temperature $T$ as an energy. 
The corresponding normal density - giving the occupancy of the particle state $i$ - is the expectation value in $|T\rangle$ of the $a_{i}^{+} a_{i}$ operator which may be written [see eq. (2.21)] as:

$$
a_{i}^{+} a_{i}=v_{i}^{2}+u_{i}^{2} \zeta_{i}^{+} \zeta_{i}-v_{i}^{2} \zeta_{\bar{i}}^{+} \zeta_{\bar{i}}+u_{i} v_{i}\left(\zeta_{i}^{+} \zeta_{\bar{i}}^{+}+\zeta_{i} \zeta_{i}\right)
$$

Consistently with the independent quasiparticle approximation (2.13) we may omit the last two terms to obtain

$$
\left\langle T\left|a_{i}^{+} a_{i}\right| T\right\rangle=n_{i}=v_{i}^{2}+\left(u_{i}^{2}-v_{i}^{2}\right) n_{i}^{T}
$$

Similarly one gets for the abnormal density

$$
\left\langle T\left|a_{i} a_{i}\right| T\right\rangle=k_{i}=u_{i} v_{i}\left(1-2 n_{i}^{T}\right) .
$$

From the expression of $u_{i}, v_{i}, n_{i}^{T}$ given in eqs. (2.9) and (2.15) one finds equivalently ${ }^{52}$ )

$$
\begin{gathered}
n_{i}=\frac{1}{2}\left[1+\frac{\lambda-\varepsilon_{i}}{\mathscr{E}_{i}} \tanh \left(\frac{\mathscr{E}_{i}}{2 T}\right)\right] \equiv n\left(\varepsilon_{i}, \lambda, \Delta_{i}\right), \\
k_{i}=\frac{\Delta_{i}}{2 \mathscr{E}_{i}} \tanh \left(\frac{\mathscr{E}_{i}}{2 T}\right) \equiv k\left(\varepsilon_{i}, \lambda, \Delta_{i}\right) .
\end{gathered}
$$

From these expressions of $n_{i}$ and $k_{i}$ one may give the $r$-representation of the normal and abnormal density matrices:

$$
\begin{aligned}
\rho\left(\boldsymbol{r}, \boldsymbol{r}^{\prime}\right) & =\sum_{i} \varphi_{i}^{*}\left(\boldsymbol{r}^{\prime}\right) \varphi_{i}(\boldsymbol{r}) n_{i}, \\
\kappa\left(\boldsymbol{r}, \boldsymbol{r}^{\prime}\right) & =\sum \varphi_{i}(\boldsymbol{r}) \varphi_{i}\left(\boldsymbol{r}^{\prime}\right) k_{i},
\end{aligned}
$$

where $\varphi_{i}(r)$ are the single-particle wave functions.

As is well known for an independent fermion equilibrium solution, the entropy is written in terms of the probabilities $n_{i}^{T}$ as

$$
S=\sum_{i} s_{i}=-\sum_{i}\left[n_{i}^{T} \ln n_{i}^{T}+\left(1-n_{i}^{T}\right) \ln \left(1-n_{i}^{T}\right)\right] .
$$

The free energy

$$
F=\langle H\rangle-T S
$$

may be written from eqs. (2.5), (2.8), (2.19) and (2.20) as

$$
F=E_{\mathrm{HFB}}+\sum_{i} \mathscr{E}_{i} n_{i}^{T}-T S+\lambda N=\operatorname{tr} \mathscr{T} \rho+\frac{1}{2} \operatorname{tr} \operatorname{tr} \rho \mathscr{V} \rho+\frac{1}{4} \operatorname{tr} \operatorname{tr} \kappa \mathscr{V} \kappa-T S,
$$


where the density matrices $\rho$ and $\kappa$ now contain the finite temperature numbers $n_{i}$ and $k_{i}$, eqs. (2.19) and (2.20), respectively.

At this point we may summarize what has been done. Starting from an effective two-body hamiltonian, we have approximated it by an independent quasiparticle hamiltonian which has served to define a canonical equilibrium at temperature $T$. The latter has been obtained by minimizing the relevant thermodynamical potential $\Omega(2.12)$. Now we have to specify the equations defining the particle and quasiparticle states. This is readily done by writing explicitly (see appendix A for the details) the variational equations in terms of functional derivatives:

$$
\frac{\delta \Omega}{\delta n_{i}^{T}}=0, \quad \frac{\delta \Omega}{\delta v_{i}^{2}}=0, \quad \frac{\delta \Omega}{\delta \varphi_{i}^{*}}=0 .
$$

The first variational equation leads, as expected, to the explicit form eq. (2.15) of the thermodynamical probabilities $n_{i}^{T}$. The second variational equation leads to the form of $v_{i}^{2}$ in eq. (2.9). The variational equation for the wave functions $\varphi_{i}^{*}$ (or $\varphi_{i}$ ) including a Lagrange multiplier for their norm - leads to the HFB equation which, using a suitable choice of the Lagrange multiplier (see appendix A) and the representation eq. (2.8), can be decoupled into the finite temperature gap equation

$$
\Delta_{i}=-\frac{1}{2} \sum_{j} k_{j}\langle i \bar{i}|\overline{\mathscr{V}}| j \bar{j}\rangle
$$

and the HF equation

$$
(\mathscr{T}+\Gamma) \varphi_{i}=\varepsilon_{i} \varphi_{i}
$$

(Note that the exchange part of the potential $\Gamma$ usually involves an integral operator!) The dependence of the solutions $\varepsilon_{i}, \varphi_{i}$ of eq. (2.28) on the temperature $T$ and the gaps $\Delta_{i}$ comes only implicitly through the density $\rho$ contained in $\Gamma$.

\section{The self-consistent Strutinsky energy theorem within the HFBT framework}

In this section, we shall derive a generalization of the so-called Strutinsky energy theorem which allows one to split up the HFBT energy into a liquid drop model (LDM) type average part and an oscillating part that contains the quantum shell fluctuations. It serves as the formal basis for the shell-correction method ${ }^{6-8}$ ), which we generalize here to a statistically excited system of quasiparticles.

In subsect. 3.1, the formal expansion of the HFBT free energy eq. (2.25) around its average part is developed to second order in the oscillating parts of the basic quantities $\rho, \kappa$ and $S$ (the entropy). In subsect. 3.2, we demonstrate how the (exact) HFBT case can be obtained from the pure HF case (non-interacting particles at 
temperature $T=0$ ) by means of an energy folding of the HF spectrum with a suitably chosen averaging function. This formal step allows us then, in subsect. 3.3, to introduce the average (LDM like) part of the HFBT free energy, and with it the averaged quantities $\tilde{\rho}, \tilde{\kappa}$ and $\tilde{S}$, in a consistent way with the help of the standard Strutinsky energy averaging technique.

An essential step not only for the rest of this section, but also for the following ones, is to show (in subsect. 3.4) that the averaging of the HFBT free energy can be done in a self-consistent way, and that the so-defined averaged energy is stationary in a variational sense. Finally, in subsect. 3.5, the energy theorem is reformulated in a way which allows one to make contact with the practical shell-correction approach, in which phenomenological LDM energies and shell-model potentials are introduced instead of the microscopically derived quantities.

\subsection{EXPANSION OF THE HFBT FREE ENERGY AROUND AN AVERAGE VALUE}

The basic idea of deriving the shell-correction method from the HF framework [see e.g. refs. $\left.{ }^{7,8}\right)$ ] consists in decomposing the density matrix $\rho$ into a statistically averaged part $\tilde{\rho}$ and an oscillating part $\delta \rho$ :

$$
\rho=\tilde{\rho}+\delta \rho
$$

A priori, the arguments do not depend on the precise definition of $\tilde{\rho}$; the main point is that the HF energy functional evaluated in terms of $\tilde{\rho}$ is supposed to determine the average part of the total HF energy in the spirit of the LDM:

$$
E_{\mathrm{HF}}[\tilde{\rho}]=\operatorname{tr} \mathscr{T} \tilde{\rho}+\frac{1}{2} \operatorname{tr} \operatorname{tr} \tilde{\rho} \tilde{\mathscr{V}} \tilde{\rho} \approx E_{\mathrm{LDM}}
$$

whereas the parts depending on $\delta \rho$ lead to the shell fluctuations. In the original articles $^{7,8}$ ) the condition (3.2) was actually taken to be the definition of $\tilde{\rho}$. Such a definition is, however, not unique; especially so since the LDM energy with its phenomenological parameters is in itself not a well-defined quantity.

For the extension to the HFBT case we have to introduce also the average parts of the abnormal density matrix $\kappa$ and of the entropy $S$ :

$$
\kappa=\tilde{\kappa}+\delta \kappa, \quad S=\tilde{S}+\delta S .
$$

We define here the density matrices $\tilde{\rho}$ and $\tilde{\kappa}$ with the help of averaged occupation numbers $\tilde{n}_{i}$ and $\tilde{k}_{i}$ :

$$
\begin{aligned}
& \tilde{\rho}\left(\boldsymbol{r}, \boldsymbol{r}^{\prime}\right)=\sum_{i} \varphi_{i}^{*}\left(\boldsymbol{r}^{\prime}\right) \varphi_{i}(\boldsymbol{r}) \tilde{n}_{i} \\
& \tilde{\kappa}\left(\boldsymbol{r}, \boldsymbol{r}^{\prime}\right)=\sum_{i} \varphi_{i}(\boldsymbol{r}) \varphi_{i}\left(\boldsymbol{r}^{\prime}\right) \tilde{k}_{i}
\end{aligned}
$$


Similarly, the averaged entropy is expressed as

$$
\widetilde{S}=\sum_{i} \tilde{s}_{i}
$$

The precise definition of the $\tilde{n}_{i}, \tilde{k}_{i}$ and $\tilde{s}_{i}$ will be given in subsect. 3.3. All we require at the moment is that they lead to average energies (or to an entropy $\tilde{S}$, respectively) that vary smoothly with nucleon numbers and with deformation.

We now use eqs. (3.1) and (3.3) to decompose the HFBT free energy (2.25) in the following way:

$$
F_{\mathrm{HFBT}}=\tilde{F}+\delta_{1} F+\delta_{2} F
$$

where

$$
\begin{gathered}
\tilde{F}=\operatorname{tr} \mathscr{T} \tilde{\rho}+\frac{1}{2} \operatorname{tr} \operatorname{tr} \tilde{\rho} \tilde{\mathscr{V}} \tilde{\rho}+\frac{1}{4} \operatorname{tr} \operatorname{tr} \tilde{\kappa} \mathscr{V} \tilde{\kappa}-T \tilde{S}, \\
\delta_{1} F=\operatorname{tr}(\mathscr{T}+\Gamma) \delta \rho-\operatorname{tr} \Delta \delta \kappa-T \delta S, \\
\delta_{2} F=-\frac{1}{2} \operatorname{tr} \operatorname{tr} \delta \rho \mathscr{V} \delta \rho-\frac{1}{4} \operatorname{tr} \operatorname{tr} \delta \kappa \widetilde{\mathscr{V}} \delta \kappa .
\end{gathered}
$$

The first quantity $\tilde{F}$ is the LDM like free energy which by construction will be free of shell effects. The first-order shell correction $\delta_{1} F(3.8)$ is the generalization of Strutinsky's shell correction $\delta_{1} E$ to $T \neq 0, \Delta_{i} \neq 0$. In terms of occupation numbers it may be written as

$$
\delta_{1} F=\sum_{i} \varepsilon_{i} \delta n_{i}-\sum_{i} \Delta_{i} \delta k_{i}-T \sum_{i} \delta s_{i}
$$

where

$$
\delta n_{i}=n_{i}-\tilde{n}_{i}, \quad \delta k_{i}=k_{i}-\tilde{k}_{i}, \quad \delta s_{i}=s_{i}-\tilde{s}_{i} .
$$

Note that with the above definitions, the decomposition of $F_{\text {HFBT }}$ eq. (3.6) is exact. The form, eq. (3.10), of the shell correction $\delta_{1} F$ is not very satisfactory, because it necessitates the explicit calculation of all the $\tilde{n}_{i}, \tilde{k}_{i}$ and $\tilde{s}_{i}$ (although this can be done consistently, of course). We shall later in sect. 3.3 derive an alternative form of $\delta_{1} F$ which is more suitable for computation.

We see from eq. (3.10) that the first-order shell correction is extracted from the following quantity:

$$
\Sigma=\operatorname{tr}(\mathscr{T}+\Gamma) \rho-\operatorname{tr} \Delta \kappa-T S=\sum_{i} \varepsilon_{i} n_{i}-\sum_{i} \Delta_{i} k_{i}-T \sum_{i} s_{i} .
$$

Note that $\Sigma$ is exactly the quantity which contains all first-order variations of $F$ as discussed in appendix A [see eq. (A.7)]. This is the generalization of the sum of 
occupied levels $\sum_{i=1}^{N} \varepsilon_{i}$, from which the shell correction is extracted in the pure HF case $\left(T=\Delta_{i}=0\right)$.

For what follows it is an essential step to demonstrate that $\Sigma$ can be obtained from the pure HF case by means of a convolution of the HF spectrum similar to the one used in the Strutinsky averaging method ${ }^{53}$ ).

\subsection{FOLDING CHARACTER OF THE ENERGY $\Sigma$}

It is in fact, possible to show that the energy $\Sigma$ can be rewritten in terms of a "HFBT level density" $g_{\mathrm{HFBT}}(E)$ as

$$
\Sigma=\int_{-\infty}^{\lambda} E g_{\mathrm{HFBT}}(E) \mathrm{d} E
$$

The HFBT level density $g_{\mathrm{HFBT}}(E)$ is obtained when replacing the $\delta$-functions in the exact HF level density

$$
g(E)=\sum_{i} \delta\left(E-\varepsilon_{i}\right)
$$

by some distributions $f_{\Delta_{i}}\left(E-\varepsilon_{i}\right)$ :

$$
g_{\mathrm{HFBT}}(E)=\sum_{i} f_{\Delta_{i} \mathrm{~T}}\left(E-\varepsilon_{i}\right)
$$

The latter are defined as

$$
f_{\Delta_{i} T}\left(E-\varepsilon_{i}\right)=\left.\frac{\partial n_{i}}{\partial \lambda}\right|_{\lambda=E}=\left.\frac{\partial}{\partial \lambda} n\left(\varepsilon_{i}, \lambda, \Delta_{i}\right)\right|_{\lambda=E},
$$

where $n_{i}$ are the HFBT occupation numbers defined in eq. (2.19). Taking the derivative in eq. (3.16), one obtains explicitly

$$
f_{\Delta_{i} T}\left(E-\varepsilon_{i}\right)=\frac{\Delta_{i}^{2}}{2 \mathscr{E}_{i}^{3}} \tanh \left(\frac{\mathscr{E}_{i}}{2 T}\right)+\frac{\left(E-\varepsilon_{i}\right)^{2}}{4 T \mathscr{E}_{i}^{2} \cosh ^{2}\left(\mathscr{E}_{i} / 2 T\right)} ;
$$

here $\mathscr{E}_{i}=\mathscr{E}\left(\varepsilon_{i}, E, \Delta_{i}\right)$ is given by eq. (2.11) replacing $\lambda$ by $E$. The chemical potential $\lambda$ is, of course, fixed by the particle number conservation:

$$
N=\int_{-\infty}^{\lambda} g_{\mathrm{HFBT}}(E) \mathrm{d} E=\sum_{i} n_{i}=\sum_{i} \int_{-\infty}^{\lambda} f_{A_{i} \mathrm{~T}}\left(E-\varepsilon_{i}\right) \mathrm{d} E
$$

With these definitions, it is just a matter of some algebra to show that the two 
TABLE 1

Some limiting cases of the HFBT approximation

(a) $T \rightarrow 0$ :

$$
\begin{aligned}
g_{\mathrm{HFBT}}(E) & \rightarrow g_{\mathrm{HFB}}(E)=\sum_{i} \frac{\Delta_{i}^{2}}{2\left[\left(E-\varepsilon_{i}\right)^{2}+\Delta_{i}^{2}\right]^{3 / 2}} \\
n_{i} & \rightarrow v_{i}^{2}=\frac{1}{2}\left[1+\frac{\left(\lambda-\varepsilon_{i}\right)}{\sqrt{\left(\lambda-\varepsilon_{i}\right)^{2}+\Delta_{i}^{2}}}\right] \\
\Sigma & \rightarrow \sum_{i} \varepsilon_{i} v_{i}^{2}-\sum_{i} \Delta_{i} u_{i} v_{i}
\end{aligned}
$$

(b) all $\Delta_{i} \rightarrow 0$ :

$$
\begin{aligned}
g_{\mathrm{HFBT}}(E) & \rightarrow g_{\mathrm{T}}(E)=\sum_{i} 4 T \frac{1}{4 T \cosh ^{2}\left(\left(E-\varepsilon_{j}\right) / 2 T\right)} \\
n_{i} & \rightarrow n_{i}^{T}=\left[1+\exp \left(\left(\varepsilon_{i}-\lambda\right) / T\right)\right]^{-1} \\
\Sigma & \rightarrow F_{\text {s.p. }}=\sum_{i} \varepsilon_{i} n_{i}^{T}-T S
\end{aligned}
$$

(c) $T$ and all $\Delta_{i} \rightarrow 0$ :

$$
\begin{aligned}
g_{\mathrm{HFBT}}(E) & \rightarrow g(E)=\sum_{i} \delta\left(E-\varepsilon_{i}\right) \\
n_{i} & \rightarrow \theta\left(\lambda-\varepsilon_{i}\right) \\
\Sigma & \rightarrow \sum_{i=1}^{N} \varepsilon_{i}
\end{aligned}
$$

The zero temperature limit, the no pairing limit and the pure HF limit are presented for the HFBT level density $g_{\mathrm{HFBT}}(E)$, the particle occupation number $n_{i}$ and the energy $\Sigma$ defined in eq. (3.12). They are readily obtained from eqs. (3.15), (3.17), (3.18) and eq. (3.12).

forms eqs. (3.13) and (3.12) of $\Sigma$ are identical. For the sake of a clearer presentation of our arguments, we give this proof in appendix $B$.

It may be interesting to study the two limiting cases $T=0$ or $\Delta_{i}=0$. The corresponding expressions for $g_{\mathrm{HFBT}}(E), n_{i}$ and $\Sigma$ are presented in table 1 ; for completeness we have also added the pure HF case.

The averaging functions for the two limiting cases which lead to the level densities $g_{\mathrm{T}}(E)$ eq. (b.1) and $g_{\mathrm{HFB}}(E)$ eq. (a.1) (shown in table 1) have been mentioned by Moretto ${ }^{35}$ ) and Vautherin ${ }^{54}$ ), respectively. Nevertheless, we think it is important to stress that in either case, the integral of $E$ times the corresponding level density according to eq. (3.13) leads to the correct variational quantity, including the heat (or pair condensation energy, respectively), from which the 1st-order shell correction $\delta_{1} F$ is to be extracted. This remains true, as shown in appendix $\mathrm{B}$, also in the most general case $T \neq 0, \Delta_{i} \neq 0$, if the averaging function $f_{\Delta_{i} T}(x)$ eq. (3.17) is used.

Two remarks are noteworthy here:

(i) Although one might think so at first sight, the function $f_{\Delta_{i} T}(x)$ is not a con- 
volution product of the two limiting functions $f_{T}(x)$ and $f_{\Delta_{i}}(x)$. This is linked to the fact that the two physical approximations leading to the HFBT scheme, namely (a) the Bogoliubov-Valatin transformation and (b) the statistics with independent quasiparticles, are not interchangeable. For our further arguments, it is however sufficient to know that the combined HFBT case can be obtained by one convolution of the HF spectrum with the function $f_{A_{i} T}(x)$.

(ii) It must be noted that $\Sigma$ contains twice the pair condensation energy $-\frac{1}{2} \operatorname{tr} \Delta \kappa$. This is natural, since the latter formally plays a similar role to that of the normal potential energy $\frac{1}{2} \operatorname{tr} \Gamma \rho$ which is also double counted in $\Sigma$. Therefore, in the limit $T=0$ the quantity $\Sigma$ does not correspond to the BCS energy usually defined in applications with a fixed one-body potential and a constant pairing matrix element $G=-\langle i \bar{i}|\overline{\mathscr{V}}| j \bar{j}\rangle$. This has some minor consequences to the practical calculations of shell corrections in the BCS framework, which we will discuss in sect. 4.

\subsection{DEFINITION OF THE AVERAGED HFBT QUANTITIES}

It has been shown in numerical HF calculations using Skyrme forces ${ }^{19-21}$ ) that the energy averaging method, introduced by Strutinsky in order to define the average part of the single-particle level density $\tilde{g}(E)$, may be used to define the averaged density matrix $\tilde{\rho}$ in a specific way, leading to average energies $E_{\mathrm{HF}}[\tilde{\rho}]$ with the desired properties as we have discussed in subsect. 3.1. We shall therefore adapt in the following the Strutinsky energy averaging to define all necessary averaged quantities.

The basic idea of the Strutinsky averaging technique is to replace the discrete single-particle level spectrum $\varepsilon_{i}$ by a continuous one which is formally obtained by a convolution of the exact level density, eq. (3.14), with an averaging function $f(x)$ :

$$
\tilde{g}(E)=\frac{1}{\gamma} \int_{-\infty}^{+\infty} g\left(E^{\prime}\right) \tilde{f}\left(\left(E-E^{\prime}\right) / \gamma\right) \mathrm{d} E^{\prime}=\frac{1}{\gamma} \sum_{i} f\left(\left(E-\varepsilon_{i}\right) / \gamma\right) .
$$

Throughout this article, the averaging function shall be understood to include the so-called curvature corrections (up to order $M$ ). The usual choice of $f(x)$ is a modified gaussian

$$
f(x)=\frac{1}{\sqrt{ } \pi} \sum_{\mu=0}^{M} \frac{(-1)^{\mu}}{2^{2 \mu} \mu !} \frac{\mathrm{d}^{2 \mu}}{\mathrm{d} x^{2 \mu}} \mathrm{e}^{-x^{2}},
$$

although this is not the only possible form, since a whole class of averaging functions exists and has been shown to lead to identical results ${ }^{53}$ ). The averaging width $\gamma$ has to be of the order of the main shell spacing of the spectrum $\varepsilon_{i}$ :

$$
\gamma \sim \hbar \omega
$$


The natural way of applying the Strutinsky averaging to the HFBT case is now to convolute $g_{\mathrm{HFBT}}(E)$ with the function $f(x)$ :

$$
\tilde{g}_{\mathrm{HFBT}}(E)=\frac{1}{\gamma} \int_{-\infty}^{+\infty} f\left(\left(E-E^{\prime}\right) / \gamma\right) g_{\mathrm{HFBT}}\left(E^{\prime}\right) \mathrm{d} E^{\prime}
$$

In terms of this averaged level density, the average part of $\Sigma$ is then defined analogously to eq. (3.13) by

$$
\tilde{\Sigma}=\int_{-\infty}^{\tilde{i}} E \tilde{g}_{\mathrm{HFBT}}(E) \mathrm{d} E,
$$

where the Fermi level $z$ is given by

$$
N=\int_{-\infty}^{\tilde{i}} \tilde{g}_{\mathrm{HFBT}}(E) \mathrm{d} E \equiv \sum_{i} \tilde{n}_{i} .
$$

It will be shown explicitly in appendix $C$ that the average quantity $\tilde{\Sigma}(3.23)$ is identical to

$$
\tilde{\Sigma}=\operatorname{tr}(\tilde{J}+\Gamma) \tilde{\rho}-\operatorname{tr} \Delta \tilde{k}-T \tilde{S}=\sum_{i}\left(\varepsilon_{i} \tilde{n}_{i}-\Delta_{i} \tilde{k}_{i}-T \tilde{s}_{i}\right),
$$

where the quantities $\tilde{\rho}, \tilde{\kappa}$ and $\tilde{s}$ are given exactly as in eqs. (3.4) and (3.5), with $\tilde{n}_{i}$, $\tilde{k}_{i}$ and $\tilde{s}_{i}$ defined by

$$
\begin{gathered}
\tilde{n}_{i}=\frac{1}{\gamma} \int_{-\infty}^{+\infty} \tilde{f}\left(\left(E-\varepsilon_{i}\right) / i\right) n\left(E, \lambda, \Delta_{i}\right) \mathrm{d} E, \\
\tilde{k}_{i}=\frac{1}{\gamma} \int_{-\infty}^{+\infty} \tilde{f}\left(\left(E-\varepsilon_{i}\right) / i\right) k\left(E, \lambda, \Delta_{i}\right) \mathrm{d} E, \\
\tilde{s}_{i}=-\frac{1}{\gamma} \int_{-\infty}^{+\infty} \tilde{f}\left(\left(E-\varepsilon_{i}\right) / \gamma\right)\left\{n_{E}^{T} \ln n_{E}^{T}+\left(1-n_{E}^{T}\right) \ln \left(1-n_{E}^{T}\right)\right\},
\end{gathered}
$$

where $n_{E}^{T}=n^{T}\left(E, \lambda, \Delta_{i}\right)$. The functions $n\left(E, \lambda, \Delta_{i}\right), k\left(E, \lambda, \Delta_{i}\right)$ and $n^{T}\left(E, \lambda, \Delta_{i}\right)$ are given by eqs. (2.19), (2.20) and (2.15), respectively, replacing $\varepsilon_{i}$ by $E$.

With these new quantities we can now express the first-order shell correction $\delta_{1} F$, eq. (3.8), as

$$
\delta_{1} F=\Sigma-\tilde{\Sigma}=\delta \Sigma
$$

which follows directly from eqs. (3.12) and (3.25). Although eq. (3.23) for $\tilde{\Sigma}$ is more 
practical to use than eq. (3.25), it is not optimal because it contains a double energy integration to be done numerically. However, as shown in appendix $\mathrm{C}$ one may find still another form of $\tilde{\Sigma}$,

$$
\tilde{\Sigma}=\int_{-x}^{+\infty} E g_{\mathrm{HFBT}}(E) \tilde{n}(E-\tilde{\lambda}) \mathrm{d} E
$$

where $\tilde{n}(E-\tilde{z})$ is the usual Strutinsky occupation number function:

$$
\tilde{n}(E-\tilde{\lambda})=\frac{1}{\gamma} \int_{-\infty}^{\tilde{\lambda}} \tilde{f}\left(\left(E-E^{\prime}\right) / \gamma\right) \mathrm{d} E^{\prime}
$$

Since both $g_{\mathrm{HFBT}}(E)$, eq. (3.15), and $\tilde{n}(E-\tilde{\pi})$ are known explicitly [the latter with eq. (3.20) in terms of a modified error function], only one single energy integration is necessary in eq. (3.28).

We emphasize that all three expressions for $\tilde{\Sigma}$, eqs. (3.23), (3.25) and (3.28), are identical only if the plateau condition

$$
\frac{\mathrm{d} \tilde{\Sigma}}{\mathrm{d} \gamma}=0
$$

is fulfilled (see appendix C). This must always be done in Strutinsky calculations to ensure the stationarity of the shell correction as a function of the averaging parameter $y$ [see e.g. ref. ${ }^{53}$ ) for a discussion of this point]. In subsect. 3.4 we will see how the plateau condition (3.30) comes about naturally in a variational formulation of the self-consistently averaged HFBT system.

With eq. (3.27) we arrive now at the following exact form of the HFBT free energy

$$
F_{\text {HFBT }}=\tilde{F}+\delta \Sigma+\delta_{2} F \text {. }
$$

This equation is one possible form of the so-called Strutinsky energy theorem [see also refs. $\left.{ }^{20,21}\right)$ ]. It is, however, not a useful one, because the shell correction $\delta \Sigma$ is here expressed in terms of the exact quantities $\varepsilon_{i}$ and $\Delta_{i}$ determined by the HFBT equations (2.27) and (2.28). In order to justify the practical shell-correction method, as pointed out by Strutinsky ${ }^{7}$ ), one must rewrite the first-order shell correction in terms of the spectrum $\hat{\varepsilon}_{i}$ of the averaged HF field $\tilde{\Gamma}$, and then show that the remaining higher-order terms are negligible. The averaged field $\tilde{\Gamma}$ may then be practically replaced by a phenomenological shell-model potential at the same time as the averaged total energy is replaced by a LDM energy [see eq. (3.2)].

Within the above framework, it is natural to define averaged fields $\tilde{\Gamma}$ and $\tilde{J}$ analogously to eq. (2.6):

$$
\begin{gathered}
\tilde{\Gamma}=\operatorname{tr} \tilde{\rho} \overline{\mathscr{V}} \\
\widetilde{J}=-\frac{1}{2} \operatorname{tr} \tilde{\kappa} \overline{\mathscr{V}}
\end{gathered}
$$


The "shell-model" spectrum $\hat{\varepsilon}_{i}$ is then defined by a HF like equation for the average field $\tilde{\Gamma}$ :

$$
(\mathscr{T}+\tilde{\Gamma}) \hat{\varphi}_{i}=\hat{\varepsilon}_{i} \hat{\varphi}_{i}
$$

In the representation $\langle\boldsymbol{r} \mid \hat{i}\rangle=\hat{\varphi}_{i}(\boldsymbol{r})$, the average gap equation (3.31) then reads

$$
\tilde{\Delta}_{i}=-\frac{1}{2} \sum_{j} \tilde{k}_{j}\langle\hat{i}|\overline{\mathscr{V}}| \hat{j}\rangle \text {. }
$$

Note that the $\tilde{k}_{j}$ also have to be calculated consistently in terms of the $\hat{\varepsilon}_{j}$ and $\tilde{J}_{j}$. As pointed out by many authors $8,17,18,20,55-57$ ), a new pair of density matrices $\hat{\rho}, \hat{\kappa}$ must be introduced in discussing the different terms of the expanded $\mathrm{HF}(\mathrm{BT})$ energy:

$$
\begin{aligned}
& \hat{\rho}\left(\boldsymbol{r}, \boldsymbol{r}^{\prime}\right)=\sum_{i} \hat{\varphi}_{i}^{*}\left(\boldsymbol{r}^{\prime}\right) \hat{\varphi}_{i}(\boldsymbol{r}) \hat{n}_{i}, \\
& \hat{\kappa}\left(\boldsymbol{r}, \boldsymbol{r}^{\prime}\right)=\sum_{i} \hat{\varphi}_{i}(\boldsymbol{r}) \hat{\varphi}_{i}^{-}\left(\boldsymbol{r}^{\prime}\right) \hat{k}_{i}
\end{aligned}
$$

The occupation numbers $\hat{n}_{i}$ and $\hat{k}_{i}$ are defined as earlier in eqs. (2.19) and (2.20), but in terms of the solutions $\hat{\varepsilon}_{i}, \tilde{J}_{i}$ of eqs. (3.32) and (3.33) (and of a new Fermi energy $\hat{\lambda}$ ).

In our earlier numerical HF calculations with Skyrme forces, we have shown ${ }^{21}$ ) that the shell-correction expansion analogous to eq. (3.6) for the HF energy (at $T=0$ ) converges best if the Strutinsky averaging of the density matrices $\tilde{\rho}$ and $\tilde{\kappa}$ eq. (3.4) is done self-consistently. (A constant phenomenological pairing matrix element $G=-\langle i \hat{i} \mid \vec{V} ! j \bar{j}\rangle$ was used in those calculations.) The self-consistency of the averaged free energy $\tilde{F}$ eq. (3.7) can be practically achieved by solving iteratively eqs. (3.32) and (3.33) and in each step recalculating the averaged densities $\tilde{\rho}$ and $\tilde{\kappa}$ in terms of the new $\hat{\varphi}_{i}, \hat{n}_{i}$ and $\hat{k}_{i}$, until convergence is reached and $\tilde{\rho} \equiv \tilde{\rho}$, $\tilde{\kappa} \equiv \tilde{\kappa}$.

Before we go on to discuss the Strutinsky energy theorem in terms of the selfconsistent spectrum, which will be done in sect. 3.5 , we shall presently prove that the self-consistently averaged HFBT system can be formulated as a variational problem.

\subsection{STATIONARITY OF THE SELF-CONSISTENTLY AVERAGED HFBT FREE ENERGY}

The variational quantity for the averaged HFBT system is its thermodynamical potential

$$
\widetilde{\Omega}=\operatorname{tr} \mathscr{T} \tilde{\rho}+\frac{1}{2} \operatorname{tr} \operatorname{tr} \tilde{\rho} \tilde{\mathscr{V}} \tilde{\rho}+\frac{1}{4} \operatorname{tr} \operatorname{tr} \tilde{\kappa} \overline{\mathscr{V}} \tilde{\kappa}-T \tilde{S}-\tilde{\lambda} \sum_{i} \tilde{n}_{i}
$$

The averaged densities $\tilde{\rho}, \tilde{\kappa}$ and occupation numbers $\tilde{n}_{i}, \tilde{k}_{i}$ and $\tilde{S}_{i}$ shall be defined 
as in the previous section, however all in terms of the solutions $\hat{\varphi}_{i}, \hat{\varepsilon}_{i}$ and $\tilde{J}_{i}$ of eqs. (3.32) and (3.33). Since we have introduced the averaging width $\gamma$ as a new parameter - the Strutinsky smoothing function $\tilde{f}(x)$ shall be fixed - we must also require the potential $\widetilde{\Omega}$ to be stationary as a function of $\gamma$ for a non-zero value of $\gamma$ [see eq. (3.21)]. This forces us to introduce a new constraint in the variation. Thus, we now show that

$$
\widetilde{\Omega}^{\prime}=\widetilde{\Omega}+\gamma \widetilde{G}
$$

is stationary with respect to all possible variations:

$$
\frac{\delta \tilde{\Omega}^{\prime}}{\delta \hat{\varphi}_{i}^{*}}=\frac{\delta \tilde{\Omega}^{\prime}}{\delta v_{i}^{2}}=\frac{\delta \tilde{\Omega}^{\prime}}{\delta n_{i}^{T}}=\frac{\delta \tilde{\Omega}^{\prime}}{\delta \gamma}=0,
$$

if we choose $\tilde{G}$ in eq. (3.36) to be

$$
\tilde{G}=\frac{\mathrm{d} \tilde{\Sigma}}{\mathrm{d} \gamma}=\sum_{i} \int_{-\infty}^{+\infty} x \tilde{f}(x) n\left(\hat{\varepsilon}_{i}+\gamma x, \tilde{\lambda}, \tilde{\Delta}_{i}\right) \mathrm{d} x .
$$

The last equality is shown in eq. (C.9) where $\tilde{\Sigma}$ is now defined by

$$
\tilde{\Sigma}=\operatorname{tr}(\mathscr{T}+\tilde{\Gamma}) \tilde{\rho}-\operatorname{tr} \tilde{\Delta} \tilde{\kappa}-T \tilde{S} \equiv \sum_{i}\left(\hat{\varepsilon}_{i} \tilde{n}_{i}-\tilde{\Delta}_{i} \tilde{k}_{i}-T \tilde{s}_{i}\right) .
$$

The variation of $\widetilde{\Omega}^{\prime}$ eq. (3.36) with respect to the wave functions $\hat{\varphi}_{i}$ (or $\hat{\varphi}_{i}^{*}$ ) goes exactly as in the non-averaged HFBT case presented in appendix A. Since the new constraint $\tilde{G}$ does not depend on the $\hat{\varphi}_{i}$, the variational equations are then

$$
\frac{\delta}{\delta \hat{\varphi}_{i}(\boldsymbol{r})}\left[\tilde{\Omega}-\tilde{\Lambda}_{i} \int\left|\hat{\varphi}_{i}(\boldsymbol{r})\right|^{2} \mathrm{~d}^{3} r\right]=0
$$

where the Lagrange multipliers $\tilde{\Lambda}_{i}$ guarantee the normalization of the $\hat{\varphi}_{i}$. As shown in appendix $\mathrm{A}$, a suitable choice of $\tilde{\Lambda}_{i}$, namely

$$
\tilde{\Lambda}_{i}=\hat{\varepsilon}_{i} \tilde{n}_{i}-\tilde{\Delta}_{i} \tilde{k}_{i}
$$

will decouple the variational equations into the HF equation (3.32) for the averaged field $\tilde{\Gamma}$ and the averaged gap equation (3.33).

Next, we have to vary the BCS occupation numbers $v_{i}^{2}$ and the thermodynamical probabilities $n_{i}^{T}$, eq. (2.15), keeping the $\hat{\varphi}_{i}$ 's and $\gamma$ fixed. In the energy representation, the total variation of $\tilde{\Omega}^{\prime}$ will be

$$
\delta \widetilde{\Omega}^{\prime}=\sum_{i}\left\{\left(\hat{\varepsilon}_{i}-\widetilde{\lambda}\right) \delta \tilde{n}_{i}-\widetilde{\Delta}_{i} \delta \tilde{k}_{i}-T \delta \tilde{s}_{i}\right\}+\gamma \delta \widetilde{G}=0 .
$$


Hereby, the variations $\delta \tilde{n}_{i}, \delta \tilde{k}_{i}$ and $\delta \tilde{s}_{i}$ individually will contain contributions from the variations $\delta v_{i}^{2}$ and $\delta n_{i}^{T}$. Before working these out, let us rewrite the explicit form of the $\tilde{n}_{i}, \tilde{k}_{i}$ and $\tilde{s}_{i}$ from eq. (3.26) after substituting $E=\gamma x+\hat{\varepsilon}_{i}$ :

$$
\begin{aligned}
& \tilde{n}_{i}=\int_{-\infty}^{+\infty} f(x) n\left(\hat{\varepsilon}_{i}+\gamma x, \lambda, \tilde{\Delta}_{i}\right) \mathrm{d} x=\int_{-\infty}^{+\infty} f(x) \hat{n}_{i}(x) \mathrm{d} x, \\
& \tilde{k}_{i}=\int_{-\infty}^{+\infty} \tilde{f}(x) k\left(\hat{\varepsilon}_{i}+\gamma x, \tilde{\lambda}_{,} \tilde{\Delta}_{i}\right) \mathrm{d} x=\int_{-\infty}^{+\infty} \tilde{f}(x) \hat{k}_{i}(x) \mathrm{d} x, \\
& \tilde{s}_{i}=\int_{-\infty}^{+\infty} \tilde{f}(x) s_{i}\left(\hat{\varepsilon}_{i}+\gamma x, \lambda, \tilde{J}_{i}\right) \mathrm{d} x=\int_{-\infty}^{+\infty} f(x) \hat{s}_{i}(x) \mathrm{d} x .
\end{aligned}
$$

The quantities $\hat{n}_{i}(x), \hat{k}_{i}(x)$ and $\hat{s}_{i}(x)$ are thus given by eqs. (2.19), (2.20) and (2.23) replacing $\varepsilon_{i}$ by $\hat{\varepsilon}_{i}+\gamma x$ and $\Delta_{i}$ by $\hat{\Delta}_{i}$. The variations of $v_{i}^{2}$ and $n_{i}^{T}$ go all through the $\hat{n}_{i}, \hat{k}_{i}$ and $\hat{s}_{i}$, which must be varied under the integrals over the smoothing function $\widetilde{f}(x)$. Using also the explicit form of $\tilde{G}$, eq. (3.38), we thus obtain

$$
\delta \tilde{\Omega}^{\prime}=\sum_{i} \int_{-\infty}^{+\infty} f(x)\left\{\left(\hat{\varepsilon}_{i}+\gamma x-\lambda\right) \delta \hat{n}_{i}(x)-\tilde{\Delta}_{i} \delta \hat{k}_{i}(x)-T \delta \hat{s}_{i}(x)\right\} \mathrm{d} x=0 .
$$

At this point, we do not need to finish explicitly the variation of $v_{i}^{2}$ and $n_{i}^{T}$. Obviously, each item in the sum of eq. (3.44) must be true independent of the choice of the Strutinsky smoothing function. Thus, the content of the curled brackets in eq. (3.44) must be zero for each $i$. However, this condition is exactly the same as in the non-averaged HFBT case for the variation of $\Omega$ with respect to $v_{i}^{2}$ and $n_{i}^{T}$, see eq. (A.7), if in the latter case $\varepsilon_{i}$ is everywhere replaced by $\hat{\varepsilon}_{i}+\gamma x$ and $\Delta_{i}$ by $\bar{J}_{i}$. [We see thus, how the choice of $\tilde{G}$; eq. (3.38), is giving exactly the extra term $\gamma \times \delta \hat{n}_{i}$ in eq. (3.44) needed for this result!] Therefore, the variations in eq. (3.44) will lead to the well-known forms of $v_{i}^{2}$, eq. (2.9), and $n_{i}^{T}$, eq. (2.15), as shown in appendix $A$, but here in terms of the $\hat{\varepsilon}_{i}, \tilde{\Delta}_{i}$ and $\tilde{\lambda}$.

We finally must ensure also that varying $\gamma$ (keeping everything else fixed) leaves $\tilde{\Omega}^{\prime}$ stationary. Now, since $\bar{\Omega}$ and $\bar{G}$ depend on $\gamma$ only through the shifted argument $\hat{\varepsilon}_{i}+\gamma x$ of the occupation numbers $\hat{n}_{i}, \hat{k}_{i}$ and $\hat{s}_{i}$ under the $x$-integration in eqs. (3.38) and (3.43), their variation with respect to $\gamma$ leads to an equation similar to eq. (3.44). Taking full account of $\gamma \widetilde{G}$ in $\widetilde{\Omega}^{\prime}$ eq. (3.36), one obtains

$$
\frac{\partial \tilde{\Omega}^{\prime}}{\partial \gamma}=\widetilde{G}+\sum_{i} \int_{-\infty}^{+\infty} \tilde{f}(x)\left\{\left(\hat{\varepsilon}_{i}+\gamma x-\tilde{\lambda}\right) \frac{\partial \tilde{n}_{i}(x)}{\partial \gamma}-\widetilde{J}_{i} \frac{\delta \tilde{k}_{i}(x)}{\partial \gamma}-T \frac{\partial \tilde{s}_{i}(x)}{\partial \gamma}\right\} \mathrm{d} x=0 .
$$

As in eq. (3.44), the content of the curly brackets will here be zero. [Although the variation is here done with respect to $\gamma$, one can verify by direct calculation, that 
this is true as a consequence of eq. (3.44).] Thus, eq. (3.45) leads to

$$
\widetilde{G}=\frac{\mathrm{d} \widetilde{\Sigma}}{\mathrm{d} \gamma}=0,
$$

which is nothing but the plateau condition (3.30) imposed on the Strutinsky averaged energy $\tilde{\Sigma}$, eq. (3.39).

In summary, we have shown that the variational principle, applied to the averaged thermodynamical potential $\tilde{\Omega}$, eq. (3.35), implies the self-consistent solution of the pair of eqs. (3.32) and (3.33), whereby in each step of the iteration the plateau condition (3.46) must be fulfilled for the Strutinsky-averaged quantity $\tilde{\Sigma}$ (3.39), as was done in the numerical calculations of ref. ${ }^{21}$ ) for the case of a constant pairing matrix element at $T=0$.

\subsection{THE STRUTINSKY ENERGY THEOREM}

We shall now rewrite the shell-correction expansion of the HFBT free energy (3.6), such that the first-order shell correction is expressed not in terms of the HFB spectrum $\varepsilon_{i}, \Delta_{i}$, but using the spectrum $\hat{\varepsilon}_{i}, \tilde{\Delta}_{i}$ of the self-consistently averaged fields $\tilde{\Gamma}$ and $\tilde{J}$ as resulting from eqs. (3.32) and (3.33). This is preferable since in the practical shell-correction approach, average (i.e., with deformation and particle numbers slowly varying) phenomenological fields are used, too.

One of the main interests of the shell-correction method is its application to the calculation of deformation energies. With the HF method these are, however, only accessible by means of external constraints, i.e. using suitable Lagrange multipliers in the HF equations [see, e.g. refs. $\left.{ }^{18,44,58}\right)$ ]. We therefore shall in the following also include such contraints. To be specific, let us constrain the (intrinsic) quadrupole moment $Q$ of the nucleus to a given value. Although a quadratic constraint is often used to obtain the convex parts of the deformation energy curve $E(Q)$, it is sufficient for the present argumentation to use a linear constraint. In the general HFBT variational treatment, we thus replace the hamiltonian $H$ by $H^{\prime}=H-\mu Q$, where $Q$ is the quadrupole moment operator and $\mu$ a Lagrange multiplier. This leads to the constrained $\mathrm{HF}$ equation,

$$
[\mathscr{T}+\Gamma-\mu q] \varphi_{i}=\varepsilon_{i} \varphi_{i}
$$

instead of eq. (2.28); here $q$ is the single-particle quadrupole operator

$$
q=3 z^{2}-r^{2} \text {. }
$$

Similarly, including a quadrupole constraint also in the variational calculation for the averaged system, we get instead of eq. (3.32)

$$
[\mathscr{T}+\tilde{\Gamma}-\tilde{\mu} q] \hat{\varphi}_{i}=\hat{\varepsilon}_{i} \hat{\varphi}_{i}
$$


The two Lagrange multipliers $\mu$ and $\tilde{\mu}$ in eqs. (3.48) and (3.49) shall be chosen such that the quadrupole moments of the two systems are equal:

$$
Q=\operatorname{tr} q \rho=\tilde{Q}=\operatorname{tr} q \tilde{\rho} .
$$

The free energies $F_{\text {HFBT }}$ and $\tilde{F}$ are, of course, still given by eqs. (2.25) and (3.7), respectively. The latter shall be understood to be obtained after solving self-consistently eq. (3.49), with the condition eq. (3.50), together with the average gap equation (3.33). Consistently, all averaged quantities like $\tilde{\rho}, \tilde{\kappa}, \tilde{S}, \tilde{n}_{i}, \tilde{k}_{i}$, etc. shall be defined in terms of the solutions $\hat{\varepsilon}_{i}, \hat{\varphi}_{i}$ and $\tilde{A}_{i}$ of these equations. (Thus, in obvious notation, we shall have $\tilde{n}_{i} \equiv \tilde{n}_{i}, \tilde{\hat{\rho}} \equiv \tilde{\rho}$, etc., due to the self-consistency.)

We now define the first-order shell correction to be

$$
\delta_{1} \hat{F}=\sum_{i}\left(\hat{\varepsilon}_{i} \delta \hat{n}_{i}-\tilde{\Delta}_{i} \delta \hat{k}_{i}-T \delta \hat{s}_{i}\right)
$$

where $\delta \hat{n}_{i}=\hat{n}_{i}-\tilde{n}_{i}$, etc. With this, the exact free energy $F_{\text {HFBT }}$ can be re-expanded to obtain

$$
F_{\mathrm{HFBT}}=\tilde{F}+\delta_{1} \hat{F}+\delta_{2} F^{\prime}+\delta_{2} F^{\prime \prime}
$$

where after a few trivial manipulations, using explicitly eq. (3.50), the last two terms have the following form:

$$
\delta_{2} F^{\prime}=\operatorname{tr}(\mathscr{T}+\Gamma)(\rho-\hat{\rho})-\operatorname{tr} \Delta(\kappa-\hat{\kappa})-T(S-\hat{S})-\mu(Q-\hat{Q}),
$$

with $\hat{Q}=\operatorname{tr} q \hat{\rho}$ and

$$
\begin{array}{r}
\delta_{2} F^{\prime \prime}=\frac{1}{2} \operatorname{tr} \operatorname{tr} \delta \rho \overline{\mathscr{V}} \delta \rho+\frac{1}{4} \operatorname{tr} \operatorname{tr} \delta \kappa \overline{\mathscr{V}} \delta \kappa-\operatorname{tr} \operatorname{tr} \delta \rho \overline{\mathscr{V}}(\rho-\hat{\rho}) \\
-\frac{1}{2} \operatorname{tr} \operatorname{tr} \delta \kappa \overline{\mathscr{V}}(\kappa-\hat{\kappa})+(\mu-\tilde{\mu})(Q-\hat{Q}) .
\end{array}
$$

The quantity $\delta_{2} F^{\prime}$ is close to zero, since it is a small variation of the constrained free energy which is stationary around the solution $(\rho, \kappa, S, Q)$. (Note that both sets of quantities $\rho, \kappa, S, Q$ and $\hat{\rho}, \hat{\kappa}, \hat{S}, \hat{Q}$ contain shell effects and that their relative differences are very small!)

The terms which contribute to $\delta_{2} F^{\prime \prime}$ are all of second order in small differences and are thus expected to be small compared to the first-order shell correction $\delta_{1} \hat{F}$. Earlier numerical experience ${ }^{20,21}$ ) shows that this is positively so for the HFlike energy parts, i.e. the terms of second order in $\delta \rho$ and $\rho-\hat{\rho}$. As to the pair condensation energy parts, there are particular situations like e.g. ${ }^{208} \mathrm{~Pb}$ in the ground state, where the exact pairing field $\Delta$ is equal to zero. In this case $\delta \kappa$ is not small compared to $\tilde{\kappa}$; the second-order terms in $\delta \kappa$ are then not negligible and must be taken explicitly into account. (Note that this is readily done in the simplified BCS 
treatment using an average pairing matrix element $G$.) This point will be discussed again in subsect. 4.2 .

The expansion eq. (3.52) of $F_{\mathrm{HFBT}}$ has the following advantages over the one given in subsect. 3.1 :

(i) The two leading terms, $\widetilde{F}$ and $\delta_{1} \hat{F}$, are determined self-consistently, i.e. the spectrum $\hat{\varepsilon}_{i}, \tilde{J}_{i}$ used to calculate the shell correction $\delta_{1} \hat{F}$ is that of the same average fields $\tilde{\Gamma}$ and $\tilde{J}$ which determine the average potential energy in $\tilde{F}$.

(ii) The stationarity with respect to the averaging width $\gamma$ is guaranteed simultaneously for $\tilde{F}$ and $\delta_{1} \hat{F}$, as shown explicitly in the previous section.

(iii) The convergence of the expansion eq. (3.52) turns out in numerical calculations (to be discussed in subsect. 4.3) to be faster than if any non-self-consistent averaging of $\tilde{F}$ is used. The self-consistent treatment of the average part thus seems to minimize the magnitude of the sum of all second-order terms $\delta_{2} F^{\prime}+\delta_{2} F^{\prime \prime}$.

Assuming the terms $\delta_{2} F^{\prime}$ and $\delta_{2} F^{\prime \prime}$ to be negligible, we thus arrive at the Strutinsky energy theorem:

$$
F_{\mathrm{HFBT}} \approx \tilde{F}+\delta_{1} \hat{F}
$$

which says that all first-order contributions of the fluctuating quantities $\delta \rho, \delta \kappa$ and $\delta S$ are contained in the quantity $\delta_{1} \hat{F}$ given by eq. (3.51).

For the practical calculation of $\delta_{1} \hat{F}$, we note that we can use the results for $\tilde{\Sigma}$ given in eqs. (3.23) or (3.28), replacing $\varepsilon_{i}, \Delta_{i}, \lambda$ by $\hat{\varepsilon}_{i}, \tilde{\Delta}_{i}, \hat{\lambda}$. We shall not discuss here the problems connected with the Strutinsky energy averaging in finite depth potentials, where the plateau condition (3.30) must be carefully observed; for a detailed discussion of this point we refer to a recent review article ${ }^{59}$ ).

The question has been raised ${ }^{55}$ ) whether the density dependence of realistic effective nucleon-nucleon interactions does not invalidate the energy theorem (3.55). This is, however, not the case. In fact, all one has to do in the above developments using a density-dependent interaction $\mathscr{V}(\rho)$, is to include the rearrangement contributions consistently in the definitions of the mean fields $\Gamma$ and $\tilde{\Gamma}$ [see also ref. $\left.{ }^{17}\right)$ ]. Thus, instead of eq. (2.6), we define

$$
\Gamma=\Gamma(\rho, \kappa)=\frac{\delta}{\delta \rho} E_{\mathrm{pot}}=\frac{\delta}{\delta \rho}\left[\frac{1}{2} \operatorname{tr} \operatorname{tr} \rho \overline{\mathscr{V}}(\rho) \rho+\frac{1}{4} \operatorname{tr} \operatorname{tr} \kappa \overline{\mathscr{V}}(\rho) \kappa\right]
$$

where the functional derivative with respect to $\rho$ must also act explicitly on $\bar{V}(\rho)$. Correspondingly, $\tilde{\Gamma}$ must be defined replacing everywhere in eq. (3.56) $\rho$ by $\tilde{\rho}$ and $\kappa$ by $\tilde{\kappa}$ :

$$
\tilde{\Gamma}=\Gamma(\tilde{\rho}, \tilde{\kappa}) .
$$

With these definitions, all the above results remain valid. 


\section{Numerical investigations on the validity of the shell-correction approach}

Deformation energy calculations using the Strutinsky approach have quickly met with a considerable success [for reviews of results, see e.g. refs. ${ }^{8,13,60-62}$ )] which may have prevented for some time a numerical study of the many assumptions involved. After the original work of Strutinsky ${ }^{6,7}$ ), numerous reformulations of his approach were given ${ }^{8,17,18,55-57}$ ) along with several numerical tests ${ }^{17-21,63-65}$ ) based on HF calculations at temperature $T=0$ (using either no pairing or BCS pairing with a constant matrix element $G$ ). Before summarizing in subsect. 4.3 the results of such tests, we will in subsect. 4.1 comment on HF calculations done at finite temperature without pairing correlations. Some problems related with the usual non-self-consistent BCS pairing approach will be discussed in subsect. 4.2. The discussion in subsect. 4.3 will be centered around numerical tests of the energy theorem eq. (3.55) and thus of the validity of the Strutinsky method in principle. It will be supplemented in subsect. 4.4 by an assessment of some of the remaining difficulties of the practical shell-correction calculations using phenomenological shell-model potentials and LDM energies.

\subsection{ON THE REDUCTION OF THE SELF-CONSISTENT APPROACH AT FINITE TEMPERATURE TO THE ZERO TEMPERATURE CASE}

In this section, we will restrict the discussion to the solution of the Hartree-Fock problem at finite temperature without pairing interactions $\left(\Delta_{i}=0\right)$. We will demonstrate that the full variational equations for the wave functions $\varphi_{i}$ and the occupation numbers $n_{i}^{T}$ need not be solved at $T \neq 0$ for practical purposes. In fact, to a very good approximation the relevant thermodynamical quantities such as excitation energy $E^{*}$ and entropy $S$ can be obtained by statistically exciting the independent fermion system defined by the self-consistent "cold" HF hamiltonian at $T=0$. This demonstration will go in two steps.

Let the solution of the HF equations (2.28) at finite temperature $T$ be characterized by the corresponding density matrix $\rho^{T}$, the HF potential $\Gamma^{T}$ and its eigenvalues $\varepsilon_{i}^{T}$. We first show that the excitation energy, defined by

$$
E^{*}(T)=E_{\mathrm{HF}}(T)-E_{\mathrm{HF}}(0),
$$

is very accurately approximated by

$$
E_{i}^{*}(T)=\sum_{i} \varepsilon_{i}^{T} n_{i}^{T}\left(\varepsilon_{i}^{T}\right)-\sum_{i=1}^{N} \varepsilon_{i}^{T},
$$

here $n_{i}^{T}\left(\varepsilon_{i}^{T}\right)$ are the usual Fermi occupation numbers [see eq. (b.2) in table 1] evaluated in terms of the $\varepsilon_{i}^{T}$. This is easily understood realizing that $E_{1}^{*}$, eq. (4.2), formally looks 
like a (negative) Strutinsky shell-correction energy; the only (but essential!) difference between the temperature smoothing (done by $n_{i}^{T}$ ) and the Strutinsky averaging (by $\tilde{n}_{i}$ ) is that the former produces the excitation of the nucleus, whereas the latter does not (due to the so-called curvature corrections). Thus, in the same way as deriving the Strutinsky energy theorem (3.52), one can show that $E_{1}^{*}$, eq. (4.2), equals the exact excitation energy (4.1) up to a small error $\Delta E_{1}^{*}$ given by

$\Delta E_{1}^{*}=E^{*}-E_{1}^{*}=\operatorname{tr}\left(\mathscr{T}+\Gamma^{0}\right)\left(\rho^{T}(0)-\rho^{0}\right)+\operatorname{tr} \operatorname{tr} \delta \rho \overline{\mathscr{V}}\left(\rho^{T}(0)-\rho^{0}\right)-\frac{1}{2} \operatorname{tr} \operatorname{tr} \delta \rho \overline{\mathscr{V}} \delta \rho$.

Here $\Gamma^{0}$ and $\rho^{0}$ are the HF potential and the density matrix, respectively, evaluated at $T=0, \delta \rho=\rho^{T}-\rho^{0}$ and $\rho^{T}(0)$ is the Slater determinant density fefined (as $\rho^{0}$ ) in terms of the eigenstates of $\Gamma^{T}$. As we argued in subsect. 3.5 for the terms $\delta_{2} F^{\prime}$ and $\delta_{2} F^{\prime \prime}$ in eq. (3.52), we can expect all terms in eq. (4.3) to be small.

Thus, for the very reasons which allow the Strutinsky expansion eq. (3.52) to converge rapidly at $T=0$ (see the numerical results in subsect. 4.3 below), the approximation $E_{1}^{*}$, eq. (4.2), for the excitation energy should be a good one. This is, in fact, confirmed numerically. In fig. 1 we show the quantity $\Delta E_{1}^{*}$, eq. (4.3), as obtained for ${ }^{208} \mathrm{~Pb}$ with the Skyrme III effective force ${ }^{66}$ ). [The HF calculations were done as described in ref. $\left.\left.{ }^{31}\right)\right]$. As can be seen, $\left|\Delta E_{1}^{*}\right|$ does not exceed $\sim 1 \mathrm{MeV}$ up to a temperature of $T \approx 4 \mathrm{MeV}$ which corresponds to a total excitation energy of $E^{*} \approx 250 \mathrm{MeV}$.

The second step is to replace the spectrum $\varepsilon_{i}^{T}$ by that of the HF potential $\Gamma^{0}$ obtained self-consistently at $T=0$, i.e. by $\varepsilon_{i}^{0}$ :

$$
E_{2}^{*}(T)=\sum_{i} \varepsilon_{i}^{0} n_{i}^{T}\left(\varepsilon_{i}^{0}\right)-\sum_{i=1}^{N} \varepsilon_{i}^{0}
$$

We claim that this remains a good approximation to the exact excitation energy. This is, in fact, due to a striking result found in extensive HF calculations at finite temperatures ${ }^{31}$ ): namely that the spectrum $\varepsilon_{i}^{T}$ is almost independent of $T$, both for light and heavy, deformed and spherical nuclei. (Their deformation, of course, being fixed with a constraint when varying $T$.) This is illustrated in fig. 2 for the case

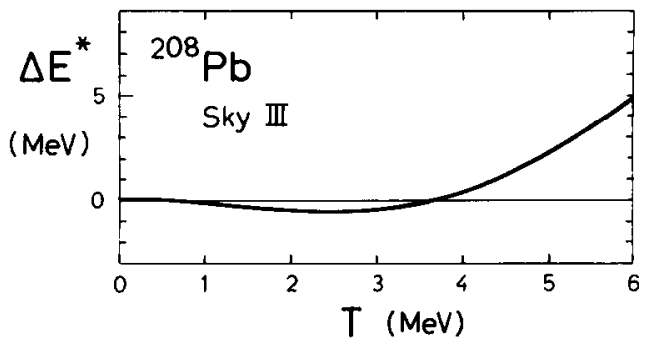

Fig. 1. Validity of the apprunimate expression (4.2) for the excitation energy. The corresponding error $\Delta E^{*}$ [see eq. (4.3)] is plotted as a function of the temperature $T$ for the ${ }^{208} \mathrm{~Pb}$ nucleus in its spherical ground state. Calculations were made with the Skyrme SIII effective force. 


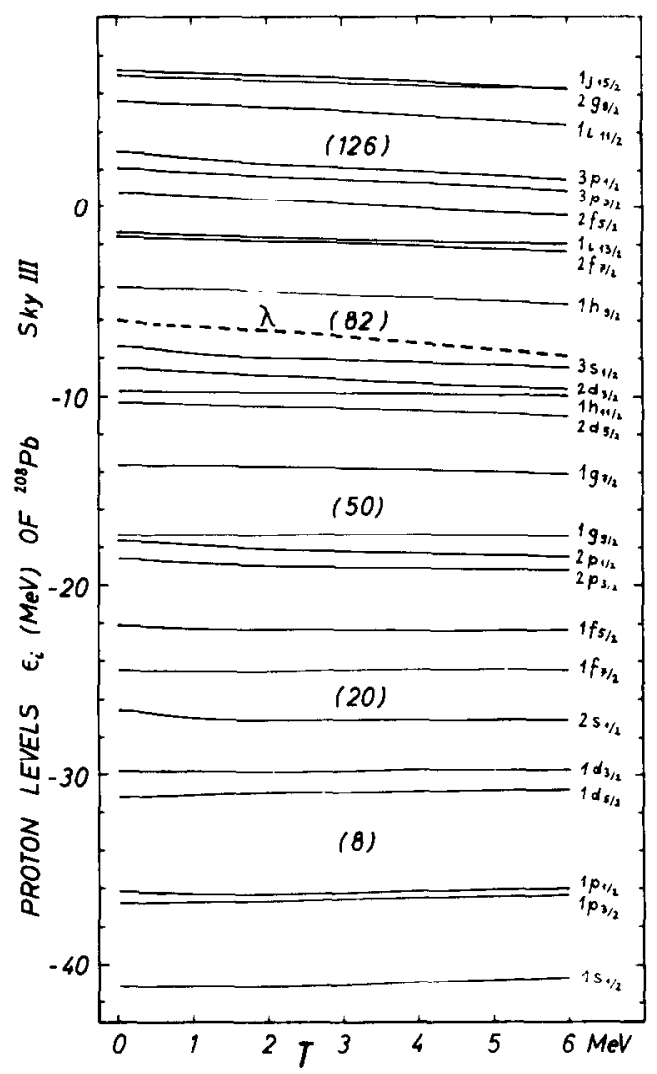

Fig. 2. Variation of the HF proton levels $\varepsilon_{i}$ as functions of the temperature $T$ ( $\lambda$ is the chemical potential). Calculations were made for the same system and with the same effective force as in fig. 1 . Numbers in brackets represent magic occupation at shell closures.

of ${ }^{208} \mathrm{~Pb}$. Between $T=0$ and $T=5 \mathrm{MeV}$, the levels $\varepsilon_{i}^{T}$ in the vicinity of the Fermi level vary by less than $\sim 1.5 \mathrm{MeV}$, also in all other cases considered. It is thus quite clear that $E_{2}^{*}$ eq. (4.4) must be a good approximation to $E_{1}^{*}$, eq. (4.2), and therefore to the exact $E^{*}$. In fact, the error $\Delta E_{2}^{*}=E^{*}-E_{2}^{*}$ can be evaluated ab initio using the same arguments as in deriving eq. (3.52) or eq. (4.3). Without explicitly assuming the constancy of $\varepsilon_{i}^{T}, \Delta E_{2}^{*}$ is found to contain only terms similar to those in eq. (4.3).

The remarkable constancy of the spectrum $\varepsilon_{i}^{T}$ as a function of $T$, together with the smallness of the terms in eq. (4.3), allow us therefore to calculate the excitation energy $E^{*}(T)$, eq. (4.1), entirely in terms of the self-consistent spectrum $\varepsilon_{i}^{0}$ of the "cold" nucleus according to eq. (4.4). The quality of this approximation has been confirmed in several numerical calculations ${ }^{31,33}$ ). In particular, if one plots the function $E^{*}(S)$ either using the self-consistent results obtained at temperature $T$, or using the approximation $E_{2}^{*}$ eq. (4.4) and the corresponding entropy $S\left(\varepsilon_{i}^{0}\right)$, the two curves are practically indistinguishable, as shown in the example of fig. 3. 


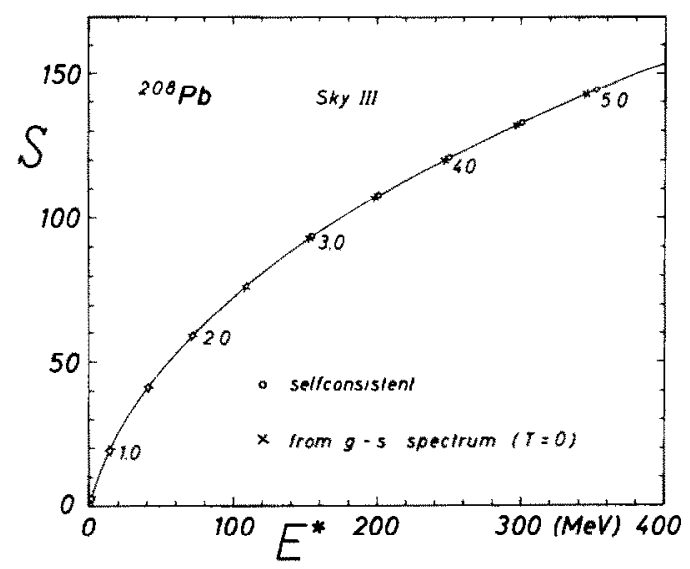

Fig. 3. Variation of the entropy $S$ as a function of excitation energy $E^{*}$. Open circles correspond to the exact HFT solutions. The temperature $T$ is varied by $0.5 \mathrm{MeV}$ steps and indicated (in $\mathrm{MeV}$ ) along the curve. Crosses represent the results obtained in terms of the $T=0$ (ground-state) levels $\varepsilon_{i}^{0}$ [see eq. (4.4)]. They are seen to lie precisely on the exact curve $S\left(E^{*}\right)$ which is almost parabolic at large $T$, as expected for a degenerate fermion system. Calculations were made for the same system and with the same effective force as in fig. 1.

In summary, we have shown that the most interesting thermodynamical quantities for excited nuclei, namely the excitation energy $E^{*}$ and the entropy $S$, can be obtained easily and accurately from the HF spectrum $\varepsilon_{i}^{0}$ of the unexcited nucleus. The full self-consistent variation of the thermodynamical potential $\Omega$ is thus not necessary; it is sufficient to statistically excite the nucleons in the "cold" HF potential. This justifies the non-self-consistent thermodynamical approach used by many groups for the calculation of nuclear level densities ${ }^{26-30}$ ).

\subsection{PAIRING CONTRIBUTIONS TO THE SHELL-CORRECTION AT ZERO TEMPERATURE}

We shall in this subsection briefly discuss the contributions of the pair correlations to the shell-correction energy and compare our present approach to other ones. We hereby restrict ourselves to the zero temperature case where most practical shellcorrection calculations have been done.

To our knowledge, the only other attempt in the literature to derive the Strutinsky energy theorem from the HFB scheme (at temperature $T=0$ ) is that of Kolomiele [ref. $\left.{ }^{25}\right)$ ]. Exploiting the formal analogy of temperature and Strutinsky averaging, he derived the following expression for the full first-order shell correction

$$
\delta_{1} E=\sum_{i} \mathscr{E}_{i} \delta v_{i}=\sum_{i} \mathscr{E}_{i}\left(v_{i}-\tilde{v}_{i}\right)
$$

where $v_{i}$ and $\tilde{v}_{i}$ are the quasiparticle occupation numbers in the exact and the averaged BCS system, respectively, and $\mathscr{E}_{i}$ are the quasiparticle energies given in eq. (2.11). 
Now, the $v_{i}$ are by definition zero, since the equation

$$
v_{i}=\left\langle 0\left|\zeta_{i}^{+} \zeta_{i}\right| 0\right\rangle=0
$$

defines the BCS ground state $|0\rangle$ as the quasiparticle vacuum. For the $\tilde{v}_{i}$, Kolomietz did not give an explicit definition, except for mentioning that care must be taken including the curvature corrections. It is therefore not obvious from ref. ${ }^{25}$ ) how to calculate explicitly the shell correction $\delta_{1} E$, eq. (4.5).

Using the formalism presented in sect. 3 , we can explicitly determine the $\tilde{v}_{i}$. Let us define the (self-consistently) Strutinsky averaged BCS ground state $|\tilde{0}\rangle$ such that, analogously to eqs. (2.3) and (2.4), we can write the averaged occupation numbers $\tilde{n}_{i}$ and the $\tilde{k}_{i},(3.4)$ and $(3.26)$, as

$$
\left\langle\tilde{0}\left|a_{i}^{+} a_{i}\right| \tilde{0}\right\rangle=\tilde{n}_{i}, \quad\left\langle\tilde{0} \mid a_{i} a_{i} \tilde{0}\right\rangle=\tilde{k}_{i} .
$$

From the Bogoliubov-Valatin transformation eq. (2.2) we find explicitly

$$
\tilde{v}_{i}=\left\langle\tilde{0}\left|\zeta_{i}^{+} \zeta_{i}\right| \tilde{0}\right\rangle=v_{i}^{2}+\left(1-2 v_{i}^{2}\right) \tilde{n}_{i}-2 u_{i} v_{i} \tilde{k}_{i}
$$

Using eqs. (4.6) and (4.8) we can now rewrite Kolomietz' shell-correction eq. (4.5) as

$$
\delta_{1} E=-\sum_{i} \mathscr{E}_{i} \tilde{v}_{i}=-\sum_{i} \mathscr{E}_{i}\left[v_{i}^{2}+\left(1-2 v_{i}^{2}\right) \tilde{n}_{i}-2 u_{i} v_{i} \tilde{k}_{i}\right]
$$

Our definition ${ }^{\dagger}$ of the shell correction according to eq. (3.10) reduces in the limit $T=0$ to

$$
\delta_{1} E=\sum_{i}\left(\varepsilon_{i} \delta n_{i}-\Delta_{i} \delta k_{i}\right)=\sum_{i}\left[\varepsilon_{i} v_{i}^{2}-\Delta_{i} u_{i} v_{i}-\varepsilon_{i} \tilde{n}_{i}+\Delta_{i} \tilde{k}_{i}\right] .
$$

Although this looks different from eq. (4.9) at first sight, it is a simple matter using eqs. (2.9) and (2.11) for $v_{i}^{2}$ and $\mathscr{E}_{i}$, to verify that both expressions for $\delta_{1} E$ are actually identical.

Thus, we have shown that the approach of Kolomietz is identical to ours in the limit $T=0$, if the above interpretation is given to the occupation numbers $v_{i}$ and $\tilde{v}_{i}$.

In all practical shell-correction calculations done so far, the pairing effects have been included using the simplified BCS approach with a constant pairing matrix element

$$
G=-\langle i \bar{i}|\overline{\mathscr{V}}| j \bar{j}\rangle
$$

+ For the moment, we ignore the difference made in sect. 3 between the spectra $\left(\varepsilon_{i}, \Delta_{i}\right)$ and $\left(\hat{\varepsilon}_{i}, \tilde{J}_{i}\right)$, since Kolomietz $\left.{ }^{25}\right)$ expresses $\delta_{1} E$ in terms of the HFB solutions $\left(\varepsilon_{i}, \Delta_{i}\right)$. 
The gaps $\Delta_{i}$ are then also constant $\left(\Delta_{i}=\Delta\right)$ and the gap equation (2.27) for $T=0$ has the familiar form

$$
\frac{1}{G}=\frac{1}{4} \sum_{i}^{\prime} \frac{1}{\mathscr{E}_{i}}=\frac{1}{4} \sum_{i}^{\prime} \frac{1}{\sqrt{\left(\varepsilon_{i}-\lambda\right)^{2}+\Delta^{2}}}
$$

(Constancy of $\Delta$ and $G$ here means independence of the state $|i\rangle$ for a fixed potential; this does not exclude, of course, a dependence of $G$ and especially of $\Delta$ on the deformation.) The primed sum in eq. (4.12) is restricted to a region $\lambda \pm \eta$ (with $\eta$ usually taken of the order of the average shell spacing $\hbar \omega$ ) for the familiar reason that it would otherwise diverge.

Our formalism in sect. 3 can easily be applied to this case taking the $\Delta_{i}$ everywhere to be

$$
\Delta_{i}= \begin{cases}\Delta & \text { for } \lambda-\eta \leqq \varepsilon_{i} \leqq \lambda+\eta \\ 0 & \text { otherwise }\end{cases}
$$

For calculating the shell correction eq. (3.51) we need the average gap $\tilde{\Delta}$ defined by eq. (3.33) which here reduces to

$$
\tilde{\Delta}=\frac{1}{2} G \sum_{i}^{\prime} \tilde{k}_{i}
$$

where the $\tilde{k}_{i}$ are given by eq. (3.26) in terms of $\tilde{\Delta}$. Using the Strutinsky averaged level density $\tilde{g}(E)$ (3.19), we can also rewrite the average gap equation (4.14) in the form

$$
\frac{1}{G}=\frac{1}{4} \int \mathrm{d} E \frac{\tilde{g}(E)}{\sqrt{(E-\bar{\lambda})^{2}+\tilde{U}^{2}}}
$$

which is analogous to eq. (4.12) for the exact gap. Both the sum and the integral in the two above equations are restricted to the energy interval $\tilde{\lambda} \pm \eta$. The definition eq. (4.15) of $\tilde{J}$ is the same as that used by Jensen and Damgaard ${ }^{26}$ ). [Also in the finite temperature limit, our definitions of the gap $\widetilde{J}$ and the entropy $\widetilde{S}$ agree with those of ref. ${ }^{26}$ ) for the case of a constant $G,(4.11)$.]

With that, the explicit contributions to the first-order shell correction coming from the pair condensation energy at $T=0$ become [see eq. (3.51)]

$$
\delta_{1} E_{\mathrm{p}}=-\operatorname{tr} \tilde{\Delta}(\hat{\kappa}-\tilde{\kappa})=-\tilde{\Delta} \sum_{i}^{\prime}\left(\hat{k}_{i}-\tilde{k}_{i}\right)=\tilde{\Delta}^{2}\left[\frac{2}{G}-\sum_{i}^{\prime} \frac{1}{2 \sqrt{\left(\hat{\varepsilon}_{i}-\hat{\lambda}^{2}+\tilde{U}^{2}\right.}}\right] .
$$

This expression differs from the one used in the usual shell-correction calculations ${ }^{7,8,13}$ ) in two ways: 
(i) As already mentioned in subsect. 3.5, our definition (4.16) only includes the terms of first order in $\delta \kappa$. In the particular cases - such as e.g. at the ground state of ${ }^{208} \mathrm{~Pb}$ - where the exact gap $\Delta$ [found from eq. (4.12)] is zero, (or very small) the terms of second order in $\delta \kappa$ [see eqs. (3.53) and (3.54)] may not be neglected. It is therefore advantageous to include them, thus replacing $\delta_{1} E_{\mathrm{p}}$, eq. (4.16), by the full difference of the exact and the average pair condensation energies:

$$
\delta E_{\mathrm{p}}=\delta_{1} E_{\mathrm{p}}+\delta_{2} E_{\mathrm{p}}=\frac{\tilde{\Delta}^{2}}{G}-\frac{\Delta^{2}}{G}
$$

This has been used in the standard shell-correction approach ${ }^{7,8,13}$ ), except for a slightly different definition of the average gap $\tilde{\Delta}$.

(ii) Instead of solving exactly the average gap equation (4.15), Strutinsky proposed ${ }^{7}$ ) to use the "uniform model" which consists in keeping $\tilde{g}(E)$ in eq. (4.15) constant at the value $\tilde{g}(\tilde{\lambda})$. The integral can then be done analytically and $G$ is chosen to yield the phenomenologically known value of $\tilde{\Delta}$ according to

$$
\bar{\Delta} \simeq 12 \mathrm{MeV} / \sqrt{ } A
$$

The total pairing shell correction (i.e. the sum of $\delta E_{\mathrm{p}}$, eq. (4.17), and the contributions contained in $\sum_{i} \hat{\varepsilon}_{i} \delta \hat{n}_{i}$ ) is then obtained in a way which is practically independent of the cut-off parameter $\Omega$ [see ref. ${ }^{8}$ ) for details]. The differences coming from the neglected energy dependence of $\tilde{g}(E)$ in the interval $\tilde{\lambda} \pm \eta$ have been tested in ref. ${ }^{26}$ ) by numerically solving eq. (4.15); they amount to less than $0.5 \mathrm{MeV}$ in the total average pairing energy in all realistic cases. As we shall see below, this difference falls well within the overall uncertainty limit of shell-correction calculations.

We conclude that along with the use of a constant pairing matrix element $G$, eq. (4.11), the inclusion of all explicit second-order pairing corrections is easy enough and numerically in accordance with the phenomenological approach by Strutinsky $\left[\right.$ refs. $\left.\left.{ }^{7,8}\right)\right]$. Numerical investigations using realistic pairing matrix elements $\langle i \bar{i}|\overline{\mathscr{V}}| j \bar{j}\rangle$ are in progress.

\subsection{NUMERICAL TESTS OF THE STRUTINSKY ENERGY THEOREM}

We will now discuss the results of extensive HF calculations at zero temperature using effective interactions of the Skyrme type ${ }^{66-68}$ ). [A short presentation of such results has appeared already in ref. ${ }^{21}$ ).] The aim of these investigations is to test the Strutinsky energy theorem eq. (3.55) (at $T=0$ ) and in particular the role of the self-consistency imposed on the averaging of the HF solutions. The pairing effects are treated in the BCS formalism with a constant pairing matrix element [see eq. (4.11)]. The full pair condensation energy correction according to eq. (4.17) is included in what below is called the first-order shell correction $\delta_{1} \hat{E}$. 
We shall demonstrate that the three quantities obtained in the shell-correction expansion of the HF energy [cf. eq. (3.52)],

$$
E_{\mathrm{HF}}=\tilde{E}+\delta_{1} \widehat{E}+\delta_{2} E
$$

have the following properties which constitute the basic assumptions of the practical shell-correction approach:

(i) The average energy $\widetilde{E}$ behaves exactly like a liquid drop (or droplet) model (LDM) energy as function of the deformation and of the nucleon numbers.

(ii) The first-order shell correction is close to the one obtained from phenomenological deformed shell-model potentials.

(iii) The sum of all second-order corrections $\delta_{2} E=\delta_{2} E^{\prime}+\delta_{2} E^{\prime \prime}$ [see eq. (3.52)] is negligible in comparison with $\delta_{1} \hat{E}$.

Let us concentrate first on the point (iii), i.e. the convergence of the expansion (4.19). In table 2 we display the energies $\widetilde{E}, \delta_{1} \hat{E}$ and $\delta_{2} E$ along with $E_{\mathrm{HF}}$ for various nuclei in their ground states, as obtained with the force Skyrme III [ref. ${ }^{66}$ )]. The smallness of $\delta_{2} E$ relative to $\delta_{1} \hat{E}$ is quite apparent. A most striking result is hereby the role played by the self-consistency of $\widetilde{E}$ and $\delta_{1} \hat{E}$ in the variational sense of subsect. 3.4. This is demonstrated with the help of the quantities $\tilde{E}_{\mathrm{NSC}}, \delta_{1} \hat{E}_{\mathrm{NSC}}$ and $\delta_{2} E_{\mathrm{NSC}}$ listed also in table 2 ; they correspond to the results obtained by applying once the Strutinsky averaging to the exact HF solution and solving only once eq. (3.32). We see that even though $\delta_{2} E_{\mathrm{NSC}}$ is reasonably small, the fully self-consistent averaging reduces the magnitude of $\delta_{2} E$ to less than $0.6 \mathrm{MeV}$ in all cases. This result is also valid as a function of deformation, as shown in fig. 4 for the example of ${ }^{240} \mathrm{Pu}$. [For further results of this kind see ref. ${ }^{21}$ ).]

The self-consistent averaging [iterating eq. (3.32)] results mainly in a redistribution of the oscillating parts of the energy; the differences between $\widetilde{E}$ and $\widetilde{E}_{\text {NSC }}$ are typically one order of magnitude smaller than $\delta_{1} \hat{E}$, as seen in table 2 (see also

TABLE 2

Self-consistent and non-self-consistent (NSC) Strutinsky energy expansion of the HF energy $\left(E_{\mathrm{HF}}\right)$ for some nuclei in their ground states, calculated with the Skyrme SIII force

\begin{tabular}{ccccccrr}
\hline Nucleus & $E_{\mathrm{HF}}$ & $\tilde{E}$ & $\delta_{1} E$ & $\delta_{2} E$ & $\tilde{E}_{\text {NSC }}$ & $\delta_{1} E_{\text {NSC }}$ & $\delta_{2} E_{\text {NSC }}$ \\
\hline${ }^{16} \mathrm{O}$ & -126.8 & -122.1 & -4.7 & 0.0 & -122.7 & -5.7 & 1.6 \\
${ }^{40} \mathrm{Ca}$ & -339.6 & -337.5 & -2.6 & 0.5 & -338.1 & -4.8 & 3.3 \\
${ }^{56} \mathrm{Ni}$ & -479.9 & -473.7 & -6.8 & 0.6 & -473.5 & -5.5 & -0.9 \\
${ }^{90} \mathrm{Zr}$ & -779.2 & -774.3 & -5.5 & 0.6 & -775.7 & -5.8 & 2.3 \\
${ }^{114} \mathrm{Sn}$ & -960.4 & -961.1 & 0.5 & 0.2 & -961.3 & 0.2 & 0.7 \\
${ }^{168} \mathrm{Yb}$ & -1352.2 & -1349.6 & -3.0 & 0.4 & -1349.9 & -4.3 & 2.0 \\
${ }^{208} \mathrm{~Pb}$ & -1625.4 & -1606.6 & -19.2 & 0.4 & -1607.1 & -20.6 & 2.3 \\
\hline
\end{tabular}

All energies are expressed in MeV. The HF energy is given as the sum $E_{\mathrm{HF}}=\tilde{E}+\delta_{1} E+\delta_{2} E$ as in eq. (3.52) in the $T=0$ limit $(F \rightarrow E)$ and defining $\delta_{2} E=\delta_{2} E^{\prime}+\delta_{2} E^{\prime \prime}$. In the ${ }^{168} \mathrm{Yb}$ case, pairing correlations have been included (i.e. $E_{\mathrm{HF}}$ stands for the $\mathrm{HF}+\mathrm{BCS}$ energy). 


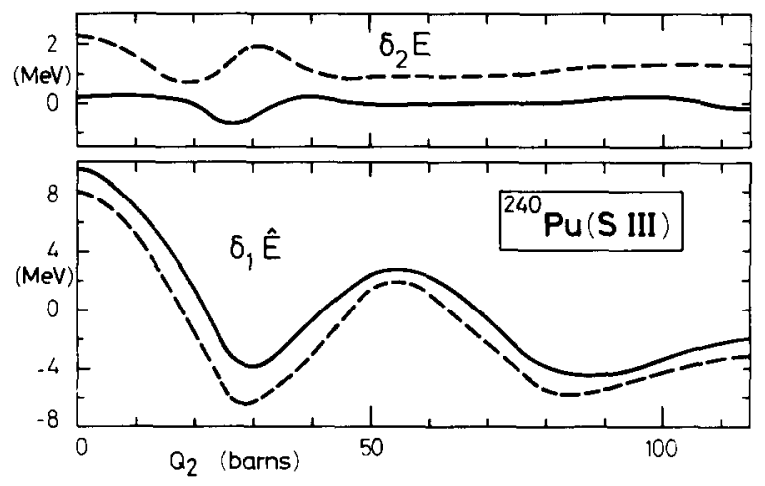

Fig. 4. First-order $\left(\delta_{1} E\right)$ and second-order $\left(\delta_{2} E\right)$ shell-correction energies along the fission barrier of ${ }^{240} \mathrm{Pu}$. Both self-consistent (full line) and non-self-consistent (dashed line) results are shown. The fission path was obtained by constraining the total quadrupole moment $Q_{2}$ (see also fig. 6). The Skyrme SIII effective force has been used.

fig. 6). We can therefore conclude that the important aspect for minimizing the term $\delta_{2} E$ is the self-consistency between the averaged potential $\tilde{\Gamma}$ and the energy $\tilde{E}$. This has some consequences for the choice of phenomenological potentials and LDM energies, as we shall see in subsect. 4.4.

In fig. 5 , a comparison is made between $\delta_{1} \hat{E}_{\mathrm{NsC}}$ calculated with two different ef-

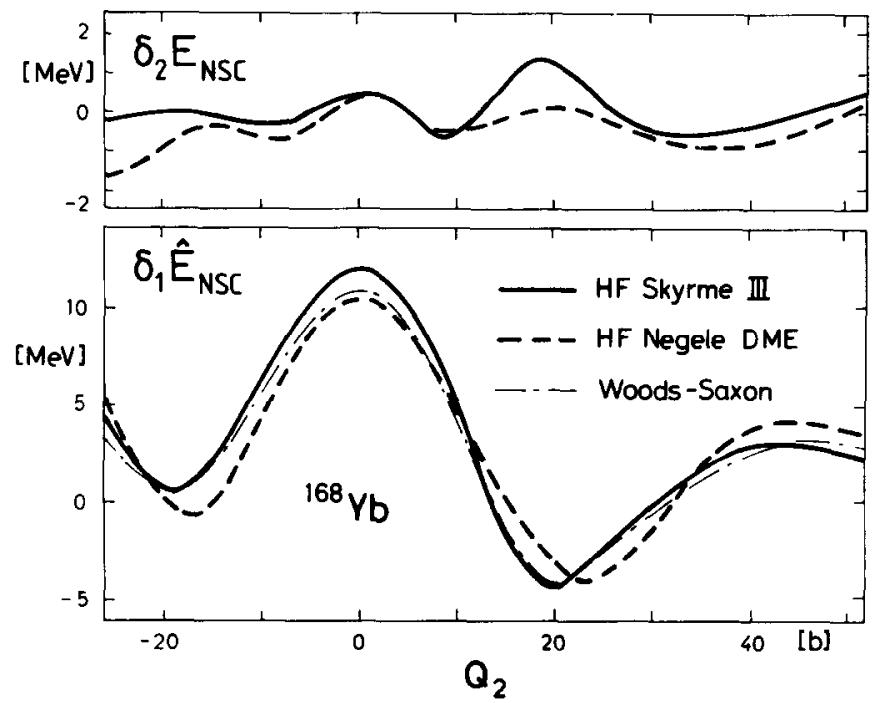

Fig. 5. First-order $\left(\delta_{1} E\right)$ and second-order $\left(\delta_{2} E\right)$ shell-correction energies along the quadrupole deformation energy curve of ${ }^{168} \mathrm{Yb}$. The results deduced from $\mathrm{HF}$ calculations are non-self-consistent and correspond to the Skyrme SIII effective force and the Negele effective force within the Negele-Vautherin ${ }^{68}$ ) density matrix expansion (DME). First-order shell corrections obtained with a Woods-Saxon parametrization ${ }^{8}$ ) of the mean field are also reported. 


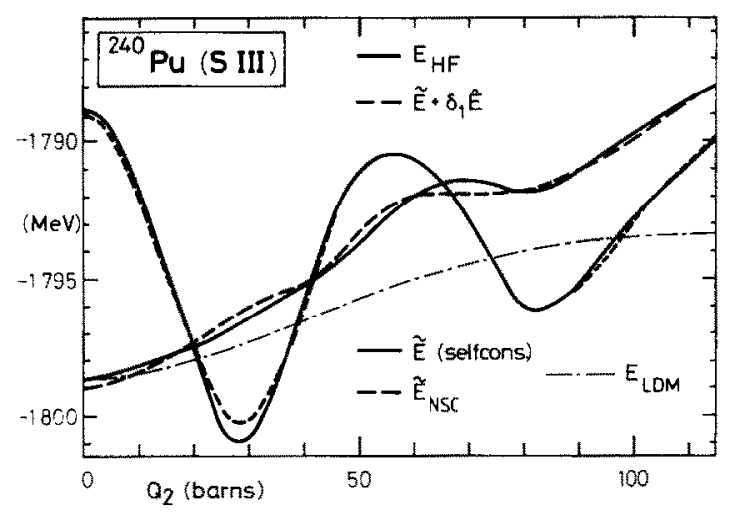

Fig. 6. Fission barrier of ${ }^{240} \mathrm{Pu}$ calculated with the Skyrme SIII effective force. The HF energy $E_{\mathrm{HF}}$ is compared with averaged energies $\tilde{E}$ (both self-consistent and non-self-consistent) and with its Strutinsky approximation $E+\delta_{1} E$. The liquid drop model ${ }^{86}$ ) estimate $E_{1 \mathrm{mM}}$ of the fission barrier is also given. The total quadrupole moment $Q_{2}$ was constrained as in fig. 4.

fective forces ${ }^{66,68}$ ) and with a phenomenological Woods-Saxon potential as used in standard shell-correction calculations ${ }^{8}$ ). The remarkable agreement between the three curves for $\delta_{1} \hat{E}_{\mathrm{NSC}}$ within $\sim 1 \mathrm{MeV}$ over the whole range of deformations strongly supports the above point (ii).

Turning to the point (i) above, the LDM like deformation behaviour of the averaged energy $\tilde{E}$ has already been demonstrated for light and medium nuclei in ref. ${ }^{21}$ ). As a further example, we show in fig. 6 the result for ${ }^{240} \mathrm{Pu}$ calculated with the force Skyrme III.

Due to the shell effects, the static fission path through the multidimensional deformation space ${ }^{\dagger}$ is generally not the same in the exact $\mathrm{HF}$ and the self-consistent average case (the non-self-consistently averaged solution follows in fact very closely the HF path). In particular, for a fixed value of the constrained quantity $Q_{2}$, the moments $Q_{4}^{\mathrm{HF}}$ and $\tilde{Q}_{4}$ determined by $\partial E_{\mathrm{HF}} / \partial Q_{4}^{\mathrm{HF}}=0$ and by $\partial \tilde{E} / \partial \widetilde{Q}_{4}=0$, respectively, are a priori not identical. Consequently, the quantity $\delta_{2} E$ calculated from eq. (4.19) spuriously contains the energy difference $\left[E_{\mathrm{HF}}\left(Q_{2}, Q_{4}^{\mathrm{HF}}\right)-E_{\mathrm{HF}}\left(Q_{2}, \tilde{Q}_{4}\right)\right]$, which is, strictly speaking, not a second-order correction. In general, however, this difference turned out to be negligible in the numerical calculations.

The results for ${ }^{240} \mathrm{Pu}$ shown in fig. 6 here constitute an exception to this general rule. Indeed, one observes some small oscillations in $\tilde{E}\left(Q_{2}\right)$ for $Q_{2} \gtrsim 50 \mathrm{~b}$ which are remnants of the fact that, whereas the equilibrium $\widetilde{Q}_{4}$ should be a smooth function of $Q_{2}, Q_{4}^{\mathrm{HF}}\left(Q_{2}\right)$ is not. For reasons of computing time limitations, we could not let the self-consistent average solution reach its equilibrium value $\widetilde{Q}_{4}$. It is easily

\footnotetext{
+ For the sake of the argument, we consider this space as being two-dimensional, assuming $Q_{4}$ to represent all multipole moments orthogonal to $Q_{2}$ which defines here the fission path.
} 
understood that the convergence rate is low in view of the small driving forces involved. This explains the oscillating behaviour of $\tilde{E}\left(Q_{2}\right)$. By inspecting the amplitude of the oscillations of $\tilde{E}\left(Q_{2}\right)$ one infers that $\left|\tilde{E}\left(Q_{2}, Q_{4}^{\mathrm{HF}}\right)-\tilde{E}\left(Q_{2}, \widetilde{Q}_{4}\right)\right|$ must not be larger than $\sim 1 \mathrm{MeV}$ in this case. Now, the difference $E_{\mathrm{HF}}\left(Q_{2}, Q_{4}^{\mathrm{HF}}\right)-E_{\mathrm{HF}}\left(Q_{2}, \widetilde{Q}_{4}\right)$ around the ${ }^{240} \mathrm{Pu} \mathrm{HF}$ ground state can be estimated from refs. ${ }^{8,108}$ ) to be $\sim 1.5$ $\mathrm{MeV}$. From our above discussion it follows that $\delta_{2} E$ is equal to $0.8 \mathrm{MeV}$ instead of $-0.7 \mathrm{MeV}$ as read from fig. 4 (see fig. 7 which illustrates this point). It is to be noted that this second-order shell-correction energy like the corresponding first-order energy $\delta_{1} \hat{E}=-3.7 \mathrm{MeV}$ ) is evaluated for the HF solution defined at the point $\left(Q_{2}, \partial_{4}\right)$, i.e. not at the HF ground state $\left(Q_{2}, Q_{4}^{\mathrm{HF}}\right)$. It is clearly seen on fig. 7 that the energy expansion (4.19) is (as already noted - see table 2) much less rapidly convergent for the non-self-consistently averaged case $\left(\delta_{1} E_{\mathrm{NSC}}=-6.5 \mathrm{MeV}\right.$ and $\left.\delta_{2} E_{\mathrm{NSC}}=1.8 \mathrm{MeV}\right)$. The latter solution, contrary to the self-consistent one, corresponds to the $\mathrm{HF}$ ground state $\left(Q_{2}, Q_{4}^{\mathrm{HF}}\right)$. It is worthwhile noting also that one extracts from fig. 6 (and 7) an energy difference $(\sim 0.3 \mathrm{MeV}) \widetilde{E}\left(Q_{2}, Q_{4}^{\mathrm{HF}}\right)-\widetilde{E}\left(Q_{2}, \widetilde{Q}_{4}\right)$ which agrees very well with the LDM result of ref. ${ }^{8}$ ).

The fact that the fission barriers turn out too high in this HF calculation with a deformation-independent pairing strength $G$ [see also ref. $\left.\left.{ }^{59}\right)\right]$ - and therefore also the average energy $\widetilde{E}$ compared to the phenomenological LDM energy shown in fig. 6 - is a defect of the effective force and of course does not invalidate our conclusions about the quality of the approximation (3.55).

The computation of $\tilde{E}$ for many different nuclei would exhibit its smooth behaviour also as a function of the nucleon numbers $N$ and $Z$ and would furthermore

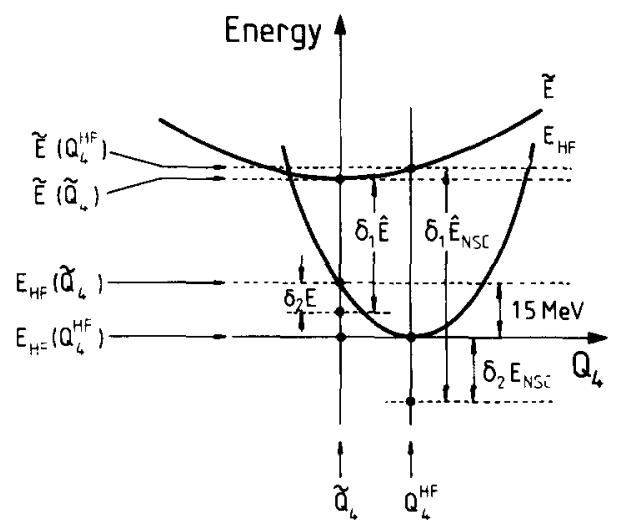

Fig. 7. Schematic hexadecapole deformation energy curves of ${ }^{240} \mathrm{Pu}$. Both $\mathrm{HF}$ energies $\left(E_{\mathrm{HF}}\right)$ and selfconsistently averaged energies $(E)$ are plotted as functions of the hexadecapole moment $Q_{4}$ (in arbitrary units), keeping the quadrupole moment $Q_{2}$ at its HF ground-state value. The relevant shell-correction energies are $\delta_{1} E=-3.7 \mathrm{MeV}, \delta_{2} E=0.8 \mathrm{MeV}, \delta_{1} E_{\mathrm{NsC}}=-6.5 \mathrm{MeV}$ and $\delta_{2} E_{\mathrm{NSC}}=1.8 \mathrm{MeV}$. The self-consistently averaged solution corresponds to the point $\left(Q_{2}, \mathscr{Q}_{4}\right)$ in the deformation surface, which is different from the HF equilibrium point $\left(Q_{2}, Q_{4}^{\mathrm{HF}}\right)$. The energy difference $E\left(Q_{2}, Q_{4}^{\mathrm{HF}}\right)-\tilde{E}\left(Q_{2}, \tilde{Q}_{4}\right)$ is equal to $0.3 \mathrm{MeV}$. The corresponding value $(-0.7 \mathrm{MeV})$ of $\delta_{2} E$ read from fig. 4 equals the present $\delta_{2} E=0.8 \mathrm{MeV}$ minus $1.5 \mathrm{MeV}$ (see text). 
allow an explicit fit of LDM parameters for a given effective force. With the microscopical method described here, this would, however, be a very time consuming task. The extraction of LDM parameters from various Skyrme-type forces has been done with a more economical, variational semiclassical method ${ }^{69}$ ) which will be discussed in subsect. 5.2 .

It has been advocated ${ }^{63}$ ) that the validity of the practical shell-correction method is contingent upon the question whether or not one has to add an external constraining field, as done in eq. (3.49), to the phenomenological shell-model potential $V_{\text {SM }}$; i.e. whether the latter should be identified with $\tilde{\Gamma}$ or with $\tilde{\Gamma}-\tilde{\mu} q$ in eq. (3.49). In ref. ${ }^{18}$ ), it was pointed out that the two sums $\sum_{i=1}^{N} \varepsilon_{i}$ and $\sum_{i=1}^{N}\left[\varepsilon_{i}-\mu q_{i}\right]$ (where $q_{i}$ are the matrix elements $\langle i|q| i\rangle$ of the quadrupole operator) behave differently, having for instance their local extrema at different deformations [see also ref. ${ }^{70}$ ) for similar results]. The fact that either of these sums show also a different deformation energy behaviour from that of the total (constrained) HF energy, was further used to raise doubts about the validity of the Strutinsky approach ${ }^{63,18}$ ). However, both these points are immaterial for the evaluation of the first-order shell correction. As we have shown in subsect. 3.5, the shell correction $\delta_{1} \hat{E}=\sum_{i} \hat{\varepsilon}_{i} \delta \hat{n}_{i}$ must a priori be evaluated from the eigenvalues $\hat{\varepsilon}_{i}$ of the averaged field including the constraint $-\tilde{\mu} q$. But the error which would be made in $\delta_{1} \hat{E}$ by leaving out the constraint is in first-order perturbation theory equal to $-\tilde{\mu} \delta Q=-\tilde{\mu}(\hat{Q}-\tilde{Q})=\tilde{\mu}(Q-\hat{Q})$ [see eq. (3.50)]. This is, however, a very small quantity - as seen in numerical calculations [refs. $\left.{ }^{19-21}\right)$ ]. It is furthermore cancelled by a corresponding term with opposite sign in the second-order correction $\delta_{2} E^{\prime \prime}$ [eq. (3.54)]. [The smallness of corrections due to the constraint has already been demonstrated in ref. ${ }^{19}$ ), fig. $3(\mathrm{~b})$.]

We also point out here that the main object of static deformation energy calculdtions are energy differences between stationary points (barrier heights etc.), at which the constraints $\mu$ and $\tilde{\mu}$ are identically zero, and are therefore not affected by the above question.

We may summarize at this point the results which have been obtained. Within the $\mathrm{HF}+\mathrm{BCS}$ framework (with constant pairing strength $G$ ), we have provided a full numerical justification of the principle of evaluating deformation energy curves at zero temperature with the usual Strutinsky method. Together with the evidence given in subsect. 4.1, we have thus also justified the currently used extension of the Strutinsky method for evaluating level densities of statistically excited nuclei [refs. $\left.{ }^{27-29}\right)$ ].

A similar test using the fully self-consistent HFB method has not been performed, but can be expected to lead to the same conclusions as those reached above because of the following reasons. First, the numerical equivalence of HFB with HF + BCS has been noted already in sect. 2. Secondly, some recent HFB calculations of the ${ }^{240} \mathrm{Pu}$ fission barrier ${ }^{50}$ ) using the Gogny force ${ }^{48.51}$ ) secm to indicatc that a HF + BCS calculation with a suitably chosen average pairing matrix element $G$ might sufficiently well reproduce the results obtained with the self-consistently calculated matrix elements $\langle i \bar{i}|\overline{\mathscr{V}}| j \bar{j}\rangle$. 


\subsection{DISCUSSION OF SOME OPEN PROBLEMS}

What we have discussed in the previous section could be phrased after Wilets a study of "the Strutinsky method as it should be". We devote the present section to some aspects of the shell-correction approach as it is actually practiced. A basic feature of the Strutinsky energy expansion as we have developed it above is its selfconsistent character [see also refs. ${ }^{57,72}$ )]. Indeed, for the validity of the reduction of $\mathrm{HFB}$ to $\mathrm{HF}+\mathrm{BCS}$, it is essential that both $\Gamma$ and $\Delta$ are derived from the same two-body interaction $\mathscr{V}$. In the pure HF case, too, it is of primary importance for the shell-correction expansion eq. (4.19) that both the average energy $\tilde{E}$ and the mean field $\tilde{\Gamma}$ generating the single-particle spectrum $\hat{\varepsilon}_{i}$ also come from the same interaction $\mathscr{r}$. This problem is further discussed in subsect. 4.4.1.

We have implicitly assumed in this paper that the mean field (HFBT or HFT + $\mathrm{BCS}$ ) approximation to the nuclear many-body problem using a phenomenological effective interaction represents the reality, i.e. that it is capable of yielding a sufficiently accurate description of deformation energies and generally of the static nuclear properties. Even though this seems a reasonable assumption in view of the broad qualitative agreement between experimental and calculated HF + BCS results [see e.g. ref. ${ }^{44}$ ) for a review], there is still room for further improvements of the effective forces in use. This will be briefly sketched in subsect. 4.4.2.

4.4.1. Anomalies in the usual shell-correction results. Shell-correction calculations starting from phenomenological mean fields have met with considerable success, particularly in describing quantitatively actinide fission barriers ${ }^{7-13,59-62}$ ). They are, however, not free from ambiguities. We will not discuss here the problems related to the energy averaging method using finite-depth potentials [since they are no real problems; see ref. ${ }^{72}$ ) and an extended discussion in ref. ${ }^{59}$ )]. We also rule out [based on our discussion in subsect. 4.3 and similar arguments in refs. ${ }^{56,57}$ )] the controversy raised about the question whether the constraining field is or is not included in the shell-model potential: the answer to it does not really matter as soon as one uses the shell-correction method properly.

A real difficulty stems from the fact that, instead of fitting once and for all a limited number of effective force parameters as in the HF approach, one has to make a separate fit of both liquid drop and shell-model parameters, the number of which is actually less limited. In doing so, there is a priori no guarantee for being consistent when combining the two ingredients. In cases where a sufficient amount of experimental data is available (as in the case of actinide fission barriers), the method is able to reproduce them very well and has even a local yet real predictive power. In less known or completely unknown regions of the nuclide chart, however, the results of shell-correction calculations should be taken with some caution. This is particularly so in the hypothetical superheavy island of stability, since the poorly known strength parameter of the spin-orbit potential crucially determines the fission stability of these nuclear species [as shown e.g. in ref. ${ }^{71}$ )]. 
The most striking illustration of the difficulties of the usual shell-correction method due to its non-self-consistent character is known as the "Pb anomaly" [refs. $\left.{ }^{8.62}\right)$ ] [which, as pointed out in ref. ${ }^{59}$ ), might be intimately related to the socalled $\mathrm{Th}$ anomaly $\left.\left.{ }^{62}\right)\right]$. Indeed, the largest discrepancy found between the results of shell-correction calculations and experimental data is the overestimation of the total binding energy of ${ }^{208} \mathrm{~Pb}$ by $\sim 4-7 \mathrm{MeV}$ when using a Woods-Saxon potential $\left[\right.$ refs. $\left.\left.{ }^{8,62}\right)\right]$. As shown in ref. ${ }^{59}$ ), this error cannot be due to the energy averaging procedure. We rather claim that it is due to an inconsistent fit of the phenomenological parameters. In fact, as shown in table 2 , it is possible to reproduce correctly the exact ${ }^{208} \mathrm{~Pb}$ binding energy in the approximation $E_{\mathrm{HF}}=\widetilde{E}+\delta_{1} \hat{E}$, whereby the energy $\tilde{E}$ has all the features of a LDM energy and the shell-correction $\delta_{1} \hat{E}$ is very close to the value obtained from a Woods-Saxon potential. Therefore, we must conclude that this $\mathrm{Pb}$ anomaly is not due to any error in $\delta_{1} \hat{E}$, but to an inconsistency between the average Skyrme energy $\widetilde{E}$ and the LDM parameters used in this case.

A similar conclusion has been reached independently by Strutinsky ${ }^{72}$ ), whereas a completely different (and seemingly orthogonal) explanation was given by Werner et al. ${ }^{73}$ ) in terms of correlations described within the Migdal theory.

The existence of such difficulties in spite of the global validity and usefulness of the shell-correction approach per se constitute an incentive for providing firmer grounds to both LDM and shell-model parametrizations. A secure and yet tractable way of doing this might be derived from semiclassical methods, as will be shown in sect. 5 . We may point out here that Strutinsky ${ }^{57.72}$ ) proposed a method of improving existing phenomenological shell-model potentials towards self-consistency which, however, has not yet been taken up in practice.

4.4.2. Some possible improvements of the effective forces in use. In order to achieve the goal of deriving self-consistently shell-model potentials and liquid drop energies, one is in need of reliable effective forces. We will discuss here possible improvements of such forces. The discussion will be restricted to Skyrme-like forces, first owing to their widespread use in many static and dynamical calculations, and secondly in view of their potential interest in self-consistent semiclassical calculations as sketched in sect. 5 .

In the last four years, the development of experimental data on giant resonances and their theoretical description in terms of RPA vibrations have yielded new constraints on effective force parametrizations. The identification of the giant isoscalar monopole resonance ${ }^{74}$ ) has led to a rather sharp determination of the incompressibility modulus of infinite nuclear matter ${ }^{75}$ ). Similarly through various expressions and approximations of RPA correlated systems, one has been able: (i) to evaluate a reasonable range for the effective nucleon mass from giant isoscalar quadrupole resonance energies ${ }^{76}$ ), (ii) to constrain the isospin symmetry properties of the effective force oul of giant isovector dipole resonance energies ${ }^{77}$ ). These experimental informations and theoretical tools not available at the time of the Skyrme force adjustment of ref. ${ }^{66}$ ) should be included in a new fitting procedure. 
One would like also to impose correct liquid drop fission barriers for actinide nuclei. Indeed it turned out from the self-consistent average HF calculations whose results are displayed in fig. 6 as well as from semiclassical calculations ${ }^{78,101}$ ) that e.g. the Skyrme SIII effective force leads to too high liquid drop fission barriers. Besides, one would like (and this is necessary for a correct treatment of the HF + BCS approximation in use here) to secure correct pairing matrix elements which has not generally been done with Skyrme-like forces. Finally, the correct assessment of the spin-orbit force strength should be done through deformed HF-BCS calculations. Indeed this would minimize the particle-vibration coupling effects on the experimental single-particle excitation energies which are compared with HF energies $\varepsilon_{i}$ in such fits. All this program is currently under completion ${ }^{110}$ ).

\section{Semiclassical models and their connections with the Strutinsky method}

\subsection{JUSTIFICATION OF THE USE OF SEMIICLASSICAL METHODS}

We shall in this section discuss the prospect of the use of semi-classical methods in solving the nuclear self-consistency problem. Whereas Hartree-Fock calculations are quite feasible for spherical nuclei using phenomenological effective nucleonnucleon interactions $48,66,67,80$ ), there is a definite need for more economical, but still self-consistent methods for the calculation of deformation energies of heavy nuclei, in particular of fission barriers. Although constrained HF calculations with different effective interactions have been successful in producing doublehumped fission barriers ${ }^{50,58,81}$ ), they require rather large computation times and cannot be used for systematic investigations on a larger scale such as is possible with the Strutinsky method. Nevertheless, the idea of determining mean fields and deformation energies of nuclei self-consistently from a given effective nucleonnucleon interaction is not only an appealing one, but is also a necessary consequence of what we have concluded in subsect. 4.4. We shall not discuss here the puzzling fact that all the above-mentioned constrained HF calculations consistently give too high fission barriers ${ }^{\dagger}$ by almost a factor 2 [see also ref. ${ }^{59}$ )], except for mentioning that if there is hope to cure this defect by a better adjustment of the effective force parameters, this gives another strong motivation for developing time saving approaches to the (deformed) self-consistency problem.

It may be questioned why semiclassical methods could be useful for deformation energy calculations, where it is obvious that the shell effects are vital to the correct description of the fission barriers. However, we have given, in sects. 3 and 4, all the evidence for why and how semiclassics may be used in this context. In fact, one of the main conclusions was that the full self-consistency is important only for the

+ Although the quadrupole moment of the isomer minimum is in agreement with experiment and the predicted spectroscopy in the second well seems reasonable [see ref. ${ }^{79}$ )]. 
averaged $\mathrm{HF}(\mathrm{BT})$ energy and the averaged fields and densities; the shell effects can be added perturbatively, after the average self-consistency has been reached, in the form of the Strutinsky shell correction evaluated from the self-consistent average potentials. In other words, the shell effects need not be present in each step of the iterative procedure, as is the case in an exact HF calculation.

Of course, our way of obtaining the averaged HF energies and potentials using the microscopical Strutinsky energy averaging is as expensive as the exact HF calculation. However, its main purpose here was to serve as a model for a semiclassical system. As a matter of fact, it is now well established that the microscopical energy averaging is completely equivalent to the so-called extended Thomas-Fermi (ETF) model which stems from a semiclassical $\hbar$-expansion of the Wigner distribution function ${ }^{82,40,83}$ ). After this equivalence has been claimed by Strutinsky ${ }^{6,7}$ ) and explicitly demonstrated by several groups ${ }^{29,40,53}$ ), the two methods have been compared very carefully for realistic potentials (including spin-orbit terms) ${ }^{41,84,85}$ ) and found to yield identical average energies within $\sim 1.5 \mathrm{MeV}$ which corresponds roughly to the error limit of either method. Therefore the Strutinsky averaged HF system discussed in sect. 3 is equivalent to a self-consistent semiclassical system. In this sense we can claim to have furnished a microscopical justification of the liquid drop model ${ }^{86}$ ) or rather the droplet model ${ }^{87}$ ). (Some caution is necessary in identifying the droplet model with a semiclassical system in our definition where "semiclassics" is equivalent to energy averaging, since the phenomenological droplet parameters are determined by least-squares fits to many nuclei. As pointed out by Strutinsky and Ivanjuk ${ }^{88}$ ), nucleon number averaging and energy averaging are not quite the same and lead to some differences particularly in shell situations with high degeneracy. [See ref. ${ }^{72}$ ) for a discussion of this point.]

The results discussed in sects. 3 and 4 provide thus a very strong motivation for the use of semiclassical models for the self-consistent calculation of average energies and potentials of deformed nuclei: if shell effects are unimportant for the average self-consistency, we should be able to arrive at it without the use of single-particle wave functions.

That the simple Thomas-Fermi approximation is not sufficient for this purpose, being unable to account for nuclear surface properties, is evident. One might therefore try to use the ETF model to express the densities $\rho(\boldsymbol{r})$ and $\tau(\boldsymbol{r})^{\dagger}$ in terms of the (HF) potential $\Gamma$ and to iterate them using a given effective force. Unfortunately, although the ETF energies obtained in the $h$-expansion are finite and converging, the corresponding densities $\rho$ and $\tau$ are not defined outside the classically allowed region; in fact, their semiclassical corrections diverge at the classical turning point. This problem has been a long-standing handicap for the direct use of semiclassical densities in the ETF model. There exist two ways out of this problem: (i) the well-

† See the definition of $\tau(\boldsymbol{r})$ in eq. (5.3). 
known and often used energy density formalism discussed in subsect. 5.2 and (ii) a newly developed "partial resummation" technique to be discussed briefly in sect. 5.3.

\subsection{ENERGY DENSITY FORMALISM AND THE HOHENBERG-KOHN THEOREM FOR SEMICLASSICAL SYSTEMS}

The idea of expressing the total binding energy $E_{\mathrm{B}}$ of the nucleus as a functional of the local density $\rho(\boldsymbol{r})$ and to formulate with it a variational principle

$$
\frac{\delta}{\delta \rho} \int \mathrm{d}^{3} r\left[\mathscr{E}_{\mathrm{B}}[\rho(\boldsymbol{r})]-\lambda \rho(\boldsymbol{r})\right]=0,
$$

has been used ever since the early days of the Fermi gas model ${ }^{89}$ ) to get simple estimates of nuclear bulk properties. Sophistication of the energy functional $\mathscr{E}_{\mathrm{B}}[\rho]$ was developed along with the understanding of the nuclear force ${ }^{90,91}$ ) and led to the so-called energy density formalism ${ }^{92,93}$ ).

The strict justification of the variational approach eq. (5.1) - apart from its intuitive appeal - was given a posteriori and came from outside nuclear physics in the form of the now famous theorem of Hohenberg and Kohn (HK) ${ }^{94}$ ). This theorem was originally formulated for a system of electrons interacting through the Coulomb force; the proof ${ }^{94}$ ) does not, however, depend explicitly on the form of the twobody force $V$ and can therefore be applied to any interacting Fermion system whose ground state is non-degenerate. For nuclear physics, the theorem thus says: the exact total ground-state energy $E_{\mathrm{B}}$ of the nucleus (including exchange and manybody correlations!) is a unique functional of the exact local ground-state one-body density $\rho(\boldsymbol{r})$. The theorem furthermore implies that the variational principle eq. (5.1) leads to the exact solutions $E_{\mathrm{B}}$ and $\rho(\boldsymbol{r})$, if the exact functional $\mathscr{E}_{\mathrm{B}}[\rho]$ is used. [Realistically, of course, one has to use separate neutron and proton densities $\rho_{\mathrm{n}}, \rho_{\mathrm{p}}$ and vary the functional $E_{\mathrm{B}}\left(\rho_{\mathrm{n}}, \rho_{\mathrm{p}}\right)$ with two Lagrange multipliers $\lambda_{\mathrm{n}}, \lambda_{\mathrm{p}}$ for the normalization to the nucleon numbers $N$ and $Z$, respectively.]

Since the exact functional $\mathscr{E}_{\mathrm{B}}[\rho]$ which depends on the nuclear two-body force is not known, one depends on models for the derivation of approximate functionals. Within the HF approximation, the problem can be put in the following form. [We shall at present stay within the pure HF limit with $T=0, \Delta_{i}=0$; the generalization of the HK theorem to $T \neq 0$ has been given by Mermin ${ }^{95}$ ); and the treatment of pairing effects will be discussed below.] We can write the HF energy as (keeping $\rho_{\mathrm{n}}=\rho_{\mathrm{p}}=\frac{1}{2} \rho$ )

$$
E_{\mathrm{HF}}=E_{\mathrm{HF}}[\rho]=T[\rho]+E_{\mathrm{pot}}[\rho]=\frac{\hbar^{2}}{2 m} \int \mathrm{d}^{3} r \tau[\rho(r)]+\int \mathrm{d}^{3} r \mathscr{E}_{\mathrm{pot}}[\rho(r)]
$$

where the kinetic energy density $\tau(r)$ is usually defined as

$$
\tau(\boldsymbol{r})=\sum_{i=1}^{N}\left|\nabla \varphi_{i}(\boldsymbol{r})\right|^{2},
$$


and is also a unique functional of $\rho(r)$. Now, it follows directly from Hohenberg and Kohn's paper ${ }^{94}$ ) that for a system of independent particles moving in a local velocity-independent potential $V(\boldsymbol{r})$, the functional $\tau[\rho]$ is not only unique but also universal, i.e. it does not depend on $V(\boldsymbol{r})$. Let us call this functional $\tau^{(0)}[\rho]$ and the corresponding kinetic energy $T^{(0)}[\rho]$. In general, the HF potential will contain also velocity-dependent terms (e.g. spin-orbit and effective mass terms); correspondingly, the kinetic energy functional must be written as

$$
T[\rho]=T^{(0)}[\rho]+T^{(\mathrm{int})}[\rho],
$$

where the part $T^{(\mathrm{int})}[\rho]$ depends explicitly on the interaction through its velocitydependent terms.

The successful use of the Skyrme-type effective interactions in HF calculations of both spherical ${ }^{66,67}$ ) and deformed nuclei ${ }^{54,58}$ ) [see also the review, ref. ${ }^{44}$ )] allows one to express the potential energy $E_{\text {pot }}$ directly as a functional of the densities $\rho(\boldsymbol{r}), \tau(\boldsymbol{r})$ and the so-called spin-orbit density ${ }^{67}$ ):

$$
J(\boldsymbol{r})=-i \sum_{i=1}^{N} \varphi_{i}^{*}(\boldsymbol{r})(\boldsymbol{\nabla} \times \boldsymbol{\sigma}) \varphi_{i}(\boldsymbol{r}) .
$$

The problem then reduces itself to the explicit determination of the functionals $\tau[\rho]$ and $J[\rho]$; if these functionals were known exactly (and we know that they do exist!), there would be no need to go over the single-particle wave functions $\varphi_{i}$ and the HF calculation could be replaced by a variational calculation of the type in eq. (5.1).

There is little hope that the exact functionals $\tau[\rho]$ and $J[\rho]$ will ever be known. However, within the ETF model these functionals can be determined as asymptotic expansions in terms of gradients of the density ${ }^{96,97}$ ) [for the part $\tau^{(0)}[\rho]$ see also ref. $\left.\left.{ }^{98}\right)\right]$ and have been successfully used in variational calculations using Skyrme forces $99,69,78$ ). In particular, the semiclassical functional for the universal part $\tau^{(0)}[\rho]$ has recently been tested extensively for local potentials using the Strutinsky averaged densities $\tilde{\rho}(\boldsymbol{r})$ and $\tilde{\tau}(\boldsymbol{r})$ [refs. $\left.{ }^{69,100}\right)$ ], and found to reproduce the semiclassical kinetic energy with excellent accuracy. [For an extensive discussion of the validity of the ETF functional $\tau^{(0)}[\rho]$ see ref. $\left.{ }^{100}\right)$.]

For the solution of the average self-consistenty problem, which is the subject of our present discussion, these semiclassical functionals appear thus as the ideal ingredients for variational calculations together with the use of Skyrme-type effective interactions. Some first calculations of average fission barriers using the ETF functionals (including spin-orbit and effective mass terms) and a restricted variational space of deformed nuclear densities have been quite promising ${ }^{78}$ ) ${ }^{\dagger}$. These studies, aiming at a thorough investigation of the applicability of Skyrme type effective forces to strongly deformed nuclei, are being further pursued ${ }^{101}$ ).

The most recent results are, in fact, in excellent agreement with the (self-consistently Strutinsky averaged) $\mathrm{HF}$ results ${ }^{111}$ ). 
The question remains whether the HK theorem can be used to justify the variational principle of eq. (5.1) using semiclassical functionals. A priori, the HK theorem holds only for the relation between the exact energy and density; can it (or a similar theorem) also be formulated for the average energy (defined in some way) as a functional of some semiclassical density? We can, based on our investigations in subsect. 3.4 above, give a positive answer to this question, at least to the extent that the selfconsistently Strutinsky averaged HF(BT) system represents a semiclassical system.

As already mentioned, the HK theorem has been generalized to the finite temperature case ${ }^{95}$ ), showing that the thermodynamical potential $\Omega$ is a unique functional of the density $\rho$ at a given temperature. Noting that the essential ingredient to the proofs in refs. ${ }^{94,95}$ ) is the fact that one starts from a variational principle, one may thus apply the same arguments to the variational system defined in subsect. 3.4, including the Strutinsky averaging in the occupation numbers, to show that the self-consistently averaged thermodynamical potential $\tilde{\Omega}^{\prime}$, eq. (3.36), is a unique functional of the average density $\tilde{\rho}(\boldsymbol{r})$ defined by

$$
\tilde{\rho}(\boldsymbol{r})=\sum_{i}\left|\hat{\varphi}_{i}(\boldsymbol{r})\right|^{2} \tilde{n}_{i}
$$

in terms of the self-consistent solutions of eqs. (3.32) and (3.33). [For an explicit discussion of the Strutinsky averaged HF case with $T=\Delta_{i}=0$, see ref. ${ }^{102}$ ).] Strictly speaking, the Strutinsky averaged $\tilde{\rho}, \tilde{\tau}$ can not be identified directly with the semiclassical ETF densities, since the latter, as stated above, are only defined inside the classically allowed region. The semiclassical functionals $\tau[\rho]$ and $J[\rho]$ derived from the ETF densities can, however, be extrapolated without problem into the classically forbidden region. The validity of this extrapolation, which has been surmised in all variational calculations using more or less sophisticated versions of the semiclassical functional $\tau[\rho]\left[\right.$ refs. $\left.\left.{ }^{69,78,89-93,99}\right)\right]$, appears to have been nicely confirmed at least numerically in the investigations of refs. ${ }^{69,100}$ ) using Strutinsky averaged densities $\tilde{\rho}$ and $\tilde{\tau}$.

Concerning the inclusion of pairing correlations, we note that Bengtsson and Schuck ${ }^{103}$ ) have recently treated pairing effects in the Thomas-Fermi approximation. From an effective force of Gogny type ${ }^{48}$ ), they derived a local density functional for the pairing condensation energy, which may be included in the energy density formalism.

\subsection{PARTIAL RESUMMATION OF THE $h$-EXPANSION}

The second method for solving the average self-consistency problem to be discussed here makes use of a newly proposed technique ${ }^{104,105}$ ) of partially resumming the semiclassical $h$-expansion. Bhaduri ${ }^{104}$ ) showed that all terms in the $h$-expansion of the single-particle Bloch density $C(r, \beta)$ which contain only first-order gradients of the potential $V(r)$ can be formally summed up and then yield a semiclassical 
density $\rho(\boldsymbol{r})$ which is well-behaved at and beyond the classical turning point. In ref. ${ }^{105}$ ) the method was extended to resum also all second-order derivatives of $V(r)$; this then is equivalent to a locally harmonic approximation of the potential $V(r)^{\dagger}$. [For the treatment of non-local potentials, see ref. $\left.{ }^{106}\right)$.] The inverse Laplace transforms, which yield the densities $\rho(\boldsymbol{r})$ and $\tau(\boldsymbol{r})$ from $C(\boldsymbol{r}, \beta)$, can be done with sufficient accuracy using the saddle point method $\left.{ }^{104,107}\right)$. The densities $\rho(r)$ and $\tau(\boldsymbol{r})$ obtained in this way after the partial resummation of $C(\boldsymbol{r}, \beta)$ are close to the ETF densities inside the classically allowed region; in the outer surface they approach the exact ones, closely following their fall-off in the tail region.

This method thus solves the long-standing turning point problem encountered in the ETF model and allows one to obtain well-behaved semiclassical densities $\rho(\boldsymbol{r})$ and $\tau(\boldsymbol{r})$ directly in terms of the potential $V(\boldsymbol{r})$ and its first two derivatives. For a Woods-Saxon potential, the energies obtained after integrating $\tau(\boldsymbol{r})$ and $V(\boldsymbol{r}) \rho(\boldsymbol{r})$ are close to the Strutinsky averaged or the ETF energies ${ }^{105,107}$ ).

Since both $\rho(\boldsymbol{r})$ and $\tau(\boldsymbol{r})$ are obtained independently with this method, they can directly be used to iterate the potential with an effective interaction without the need of a functional relation $\tau[\circ]$. This iteration procedure is thus analogous to the HF iterational method, but without the use of wave functions. Its realization for spherical nuclei is in progress ${ }^{107}$ ) and is hoped soon to yield self-consistent semiclassical nuclear binding energies and potentials.

\subsection{DOING HF WITHOUT REALLY DOING IT}

As a natural conclusion to this section we may stress again that starting from an effective hamiltonian, there exist cheap ways of producing deformation energy curves without going through heavy numerical calculations. The self-consistency is needed only for the semiclassical part of the solution. The latter provides a shellmodel potential $\tilde{\Gamma}$ which can be used in two ways to evaluate accurately the shell effects: (i) by the shell-correction method discussed at length here, (ii) by the socalled expectation value method ${ }^{108,109}$ ) which consists in evaluating the HF energy as the expectation value of the effective hamiltonian for the solution of the one-body Schrödinger equation associated to $\mathscr{T}+\tilde{\Gamma}$. The former method has been shown in subsect. 4.3 to yield very accurately the HF total energy insofar as the semiclassical calculation has been performed with a sufficient accuracy. The expectation value method, in turn, has the advantage of perturbatively curing possible deficiencies of the semiclassical treatment [as can be deduced e.g. from the results of ref. ${ }^{99}$ )] along with adding the shell effects.

\footnotetext{
${ }^{\dagger}$ See also ref. ${ }^{43}$ ).
} 


\section{Summary}

We have given a unified, self-consistent description of the Strutinsky theory within the Hartree-Fock-Bogoliubov framework at finite temperature (HFBT), which allows one to explicitly calculate the shell corrections for an excited system of quasiparticles. Our definition of the relevant average quantities is consistent with two earlier approaches in the limit of a constant pairing matrix element $G$ and the zero-temperature limit.

Supported by numerical HF results, we demonstrated first how the self-consistent finite-temperature treatment can be reduced to one at zero temperature, and secondly that the HF + BCS energy can be decomposed into an (self-consistent) average liquid drop like energy plus the usual Strutinsky shell correction.

An essential feature of the averaged HFBT solution is its variational character. This allowed us to argue that the Hohenberg-Kohn theorem should hold for the selfconsistently averaged HFBT system and how this justifies the use of semiclassical methods; two of them were shown to be promising economical tools for the solution of the average nuclear self-consistency problem.

The authors are indebted to Dr. B. Jennings for a critical reading of various parts of the manuscript and for very stimulating discussions. We also acknowledge the hospitality extended to us by each other's institutions during several mutual visits. Some of the numerical calculations have been performed at the IPN, Division of Theoretical Physics at Orsay. We thank its computer staff for excellent working conditions.

\section{Appendix A}

STATIONARITY OF THE THERMODYNAMICAL POTENTIAL $\Omega$

We shall demonstrate that the thermodynamical potential $\Omega$ [see eqs. (2.12), (2.25)],

$$
\Omega=\operatorname{tr}\left(\mathscr{T}+\frac{1}{2} \Gamma\right) \rho-\frac{1}{2} \operatorname{tr} \Delta \kappa-T S-\lambda N,
$$

is stationary with respect to individual variations of (a) the single-particle wave functions $\varphi_{i}$, (b) the BCS occupation numbers $v_{i}^{2}$ and (c) the thermodynamical occupation numbers $n_{i}^{T}$, eq. (2.15).

(a) The variation of the $\varphi_{i}(\boldsymbol{r})$ (or $\varphi_{i}^{*}(\boldsymbol{r})$ ) is done in $r$-space. Since the terms $T S$ and $\lambda N$ in eq. (A.1) do not depend explicitly on the wave functions, the variational equations for the $\varphi_{i}^{*}(\boldsymbol{r})$ are, after including Lagrange multipliers $\underline{\Lambda_{i}}$ which ensure their normalization:

$$
\frac{\delta}{\delta \varphi_{i}^{*}(r)}\left[\operatorname{tr}\left(\mathscr{T}+\frac{1}{2} \Gamma\right) \rho-\frac{1}{2} \operatorname{tr} \Delta \kappa-\Lambda_{i} \int\left|\varphi_{i}(r)\right|^{2} \mathrm{~d}^{3} r\right]=0 .
$$


With the explicit forms of $\Gamma, \Delta, \rho$ and $\kappa$ given in sect. 2, the variations (A.2) lead to the following set of $\operatorname{HFB}(\mathrm{T})$ equations

$$
(\mathscr{T}+\Gamma) \varphi_{i} n_{i}-\Delta \varphi_{i}^{*} k_{i}=\Lambda_{i} \varphi_{i}
$$

(Variation of the $\varphi_{i}$ leads, after complex conjugation, to the same equations.) The temperature dependence only comes in here implicitly through the occupation numbers.

Eqs. (A.3) couple the states $\varphi_{i}$ to their time-reversed and complex conjugated states $\varphi_{i}^{*}$. (Note that the pairing field $\Delta$ and, in general, the exchange part of the potential $\Gamma$ are non-local operators, involving an integration over the wave functions to their right side.) However, with a suitable choice of the Lagrange multipliers $\Lambda_{i}$, eqs. (A.3) can be used to decouple the gap equations (2.27) on one hand and the HF equations (2.28) for the potential $\Gamma$ on the other hand. This is shown by multiplying eq. (A.3) from the left with $\varphi_{j}^{*}$ and integrating over space. One obtains then

$$
(\mathscr{T}+\Gamma)_{j i} n_{i}-\Delta_{j i}^{*} k_{i}=\Lambda_{i} \delta_{i j}
$$

Using the representation (2.8) $\left(\Delta_{i j}^{*}=\Lambda_{i} \delta_{i j}\right)$ and choosing

$$
\Lambda_{i}=\varepsilon_{i} n_{i}-\Delta_{i} k_{i}
$$

eq. (A.4) then leads to eq. (2.28).

(b) The variation of the $v_{i}^{2}$ and $n_{i}^{T}$ is most easily done in the HFBT representation (2.8), where $\Omega$ reads

$$
\Omega=\sum_{i}\left\{\left[\mathscr{T}_{i}+\frac{1}{2} \Gamma_{i}\right] n_{i}-\lambda n_{i}-\frac{1}{2} \Delta_{i} k_{i}-T S_{i}\right\}
$$

The full variation of $\Omega$ then gives

$$
\delta \Omega=\sum_{i}\left\{\left(\varepsilon_{i}-\lambda\right) \delta n_{i}-\Delta_{i} \delta k_{i}-T \delta s_{i}\right\}=0,
$$

whereby the $\delta n_{i}, \delta k_{i}$ and $\delta s_{i}$ receive contributions from varying separately $v_{i}^{2}$ and $n_{i}^{T}$. Of course, each item in the sum in eq. (A.7) must be separately zero. In varying the $v_{i}^{2}$ and $n_{i}^{T}$, we use the explicit forms $n_{i}$, eq. (2.17), $k_{i}$, eq. (2.18), and $s_{i}$, eq. (2.23).

Varying $v_{i}^{2}$ with $u_{i}^{2}=1-v_{i}^{2}$ leads to (note that $\partial s_{i} / \partial v_{i}^{2}=0$ !)

$$
\left(\varepsilon_{i}-\lambda\right)\left(1-2 n_{i}^{T}\right)-\Delta_{i} \frac{\left(u_{i}^{2}-v_{i}^{2}\right)}{2 u_{i} v_{i}}\left(1-2 n_{i}^{T}\right)=0 .
$$

The factor $\left(1-2 n_{i}^{T}\right)$ splits off, and the rest of eq. (A.8) leads to the explicit form of $v_{i}^{2}$ eq. (2.9). 
Varying $n_{i}^{T}$ gives finally

$$
\left(\varepsilon_{i}-\lambda\right)\left(u_{i}^{2}-v_{i}^{2}\right)+2 \Delta_{i} u_{i} v_{i}+T \ln \left[n_{i}^{T} /\left(1-n_{i}^{T}\right)\right]=0 .
$$

Using the result (2.9) for $u_{i}$ and $v_{i}$, one obtains with eq. (2.11)

$$
\mathscr{E}_{i}=T \ln \left[\left(1-n_{i}^{T}\right) / n_{i}^{T}\right]
$$

which leads to eq. (2.15) for the $n_{i}^{T} s$.

\section{Appendix B}

PROOF OF EQ. (3.13) FOR $\Sigma$

Inserting the HFBT level density eq. (3.15) into eq. (3.13), subtracting and adding $\sum_{i} \varepsilon_{i} n_{i}$ we get using (eq. (3.18)),

$$
\Sigma=\sum_{i} \int_{-\infty}^{\lambda}\left(E-\varepsilon_{i}\right) f_{A_{i} T}\left(E-\varepsilon_{i}\right) \mathrm{d} E+\sum_{i} \varepsilon_{i} n_{i}=\sum_{i} F_{i}+\sum_{i} \varepsilon_{i} n_{i} .
$$

We now integrate $F_{i}$ once by parts using eq. (3.16) to obtain

$$
F_{i}=\left(\lambda-\varepsilon_{i}\right) n_{i}-\int_{-\infty}^{\lambda} n\left(\varepsilon_{i}, E, \Delta_{i}\right) \mathrm{d} E
$$

The second term can be integrated directly using the explicit form (2.19) of $n\left(\varepsilon_{i}, E, \Delta_{i}\right)$. As a result we get for $F_{i}$

$$
F_{i}=\frac{\left(\lambda-\varepsilon_{i}\right)^{2}}{2 \mathscr{E}_{i}} \tanh \left(\frac{\mathscr{E}_{i}}{2 T}\right)-T \ln \left[2 \cosh \left(\frac{\mathscr{E}_{i}}{2 T}\right)\right] .
$$

Noting that the entropy $S$, eq. (2.23), can also be written as

$$
S=\sum_{i} s_{i}=-\sum_{i} \frac{\mathscr{E}_{i}}{2 T} \tanh \left(\frac{\mathscr{E}_{i}}{2 T}\right)+\sum_{i} \ln \left[2 \cosh \left(\frac{\mathscr{E}_{i}}{2 T}\right)\right],
$$

and using the form (2.20) of $k_{i}$ we see thus that

$$
F_{i}=-T s_{i}-\Delta_{i} k_{i}
$$

This, together with the r.h.s. of eq. (B.1), leads to the definition, eq. (3.12), of $\Sigma$. 


\section{Appendix C}

PROOF OF EQS. (3.25) AND (3.28) FOR $\tilde{\Sigma}$

The definition of $\tilde{\Sigma}$, eq. (3.23), gives, with eqs. (3.22) and (3.15),

$$
\tilde{\Sigma}=\sum_{i} \frac{1}{\gamma} \int_{-\infty}^{\tilde{\lambda}} E \mathrm{~d} E \int_{-\infty}^{+\infty} \mathrm{d} E^{\prime} \tilde{f}\left(\left(E-E^{\prime}\right) / \gamma\right) f_{\Delta_{i} T}\left(E^{\prime}-\varepsilon_{i}\right) .
$$

After substituting $E^{\prime}=E-\gamma x$ and using the fact that $f^{\prime}(x)$ is an even function, we get

$$
\tilde{\Sigma}=\sum_{i} \int_{-\infty}^{+\infty} \tilde{f}(x) \mathrm{d} x \int_{-\infty}^{\tilde{\lambda}} E f_{\Delta_{i} \mathrm{~T}}\left(E-\gamma x-\varepsilon_{i}\right) \mathrm{d} E=\int_{-\infty}^{+\infty} \tilde{f}(x) \mathrm{d} x \Sigma(x),
$$

where $\Sigma(x)$ is defined exactly as $\Sigma$, eq. (3.13), if we there replace $\varepsilon_{i} \rightarrow \varepsilon_{i}+\gamma x$ and $\lambda \rightarrow \lambda$. We can therefore immediately use the form of $\Sigma$, eq. (3.12), to write with the same replacements

$$
\tilde{\Sigma}=\int_{-\infty}^{+\infty} \tilde{f}(x) \mathrm{d} x \sum_{i}\left\{\left(\varepsilon_{i}+\gamma x\right) n_{i}(x)-\Delta_{i} k_{i}(x)-T s_{i}(x)\right\},
$$

where

$$
\begin{gathered}
n_{i}(x)=n\left(\varepsilon_{i}+\gamma x, \tilde{\lambda}, \Delta_{i}\right), \\
k_{i}(x)=k\left(\varepsilon_{i}+\gamma x, \bar{\lambda}, \Delta_{i}\right), \\
s_{i}(x)=-\left[n_{i}^{T}(x) \ln n_{i}^{T}(x)+\left(1-n_{i}^{T}(x)\right) \ln \left(1-n_{i}^{T}(x)\right)\right], \\
n_{i}^{T}(x)=n^{T}\left(\varepsilon_{i}+\gamma x, \tilde{\lambda}, \Delta_{i}\right),
\end{gathered}
$$

thus using the definitions of eqs. (2.15), (2.19) and (2.20) with the shifted arguments. Doing the $x$-integrations in eq. (C.3) under the sum, one obtains

$$
\tilde{\Sigma}=\sum_{i}\left(\varepsilon_{i} \tilde{n}_{i}-\Delta_{i} \tilde{k}_{i}-T \tilde{s}_{i}\right)+\gamma \int_{-\infty}^{+\infty} x \tilde{f}(x)\left(\sum_{i} n_{i}(x)\right) \mathrm{d} x,
$$

whereby the occupation numbers $\tilde{n}_{i}, \tilde{k}_{i}$ and the $\tilde{s}_{i}$ are defined as in eq. (3.26). To recognize the relevance of the last term in eq. (C.6), we calculate the total derivative of $\tilde{\Sigma}$ with respect to $\gamma$ from eqs. (3.23) and (3.24):

$$
\frac{\mathrm{d} \tilde{\Sigma}}{\mathrm{d} \gamma}=\int_{-\infty}^{\tilde{\lambda}}(E-\tilde{\lambda}) \frac{\mathrm{d}}{\mathrm{d} \gamma} \tilde{g}_{\mathrm{HFBT}}(E) \mathrm{d} E .
$$


From eqs. (3.22) and (3.15), we find, substituting again $E^{\prime}=E-\gamma x$,

$$
\frac{\mathrm{d}}{\mathrm{d} \gamma} \tilde{g}_{\mathrm{HFBT}}(E)=\int_{-\infty}^{+\infty} \tilde{f}(x) \mathrm{d} x \sum_{i}(-x) \frac{\mathrm{d}}{\mathrm{d} E} f_{\Delta_{i} T}\left(E-\gamma x-\varepsilon_{i}\right) .
$$

Inserting eq. (C.8) into eq. (C.7), we get after partial integration over $E$ and using eq. (3.16)

$$
\frac{\mathrm{d} \tilde{\Sigma}}{\mathrm{d} \gamma}=\sum_{i} \int_{-\infty}^{+\infty} \tilde{f}(x) x n\left(\varepsilon_{i}+\gamma x, \lambda, \Delta_{i}\right) \mathrm{d} x .
$$

This gives for $\tilde{\Sigma}$, with eqs. (C.4) and (C.6),

$$
\tilde{\Sigma}=\sum_{i}\left(\varepsilon_{i} \tilde{n}_{i}-\Delta_{i} \tilde{k}_{i}-T \tilde{s}_{i}\right)+\gamma \frac{\mathrm{d} \tilde{\Sigma}}{\mathrm{d} \gamma},
$$

a formula which is consistent with the one for the case $T=0, \Delta_{i}=0$ derived in ref. ${ }^{53}$ ).

Now, when Strutinsky averaging one wants to obtain results that are (at least locally) independent of the averaging parameter $\gamma$. Thus, we use here, too, the plateau condition

$$
\frac{\mathrm{d} \tilde{\Sigma}}{\mathrm{d} \gamma}=0
$$

which then leads to the results $(3.25)$ for $\tilde{\Sigma}$.

To obtain the alternative form of $\tilde{\Sigma}$, eq. (3.28), we start from eq. (C.1) again. Adding and subtracting the same quantity, we can rewrite $\tilde{\Sigma}$ as

$$
\begin{aligned}
\tilde{\Sigma}=\sum_{i} \frac{1}{\gamma} \int_{-\infty}^{+\infty} \mathrm{d} E^{\prime} \int_{-\infty}^{\tilde{\lambda}}(E & \left.-E^{\prime}\right) \tilde{f}\left(\left(E-E^{\prime}\right) / \gamma\right) f_{\Delta_{i} \mathrm{~T}}\left(E^{\prime}-\varepsilon_{i}\right) \mathrm{d} E \\
& +\sum_{i} \frac{1}{\gamma} \int_{-\infty}^{+\infty} E^{\prime} f_{\Delta_{i} \mathrm{~T}}\left(E^{\prime}-\varepsilon_{i}\right) \mathrm{d} E^{\prime} \int_{-\infty}^{\tilde{\lambda}} \tilde{f}\left(\left(E-E^{\prime}\right) / \gamma\right) \mathrm{d} E .
\end{aligned}
$$

The first term in eq. (C.12), after substituting $E-E^{\prime}=\gamma x$, is recognized as $\gamma \mathrm{d} \tilde{\Sigma} / \mathrm{d} \gamma$ with eq. (C.9), and thus is vanishing. The second term, with eqs. (3.15) and (3.29), gives then the result eq. (3.28) for $\widetilde{\Sigma}$.

\section{References}

1) S. G. Nilsson, Mat. Fys. Medd. Dan. Vid. Selsk. 29 (1955) no. 16

2) B. R. Mottelson and S. G. Nilsson, Mat. Fys. Medd. Dan. Vid. Selsk. 32 (1961) no. 16

3) S. A. E. Johansson, Nucl. Phys. 12 (1959) 449; 22 (1962) 529 
4) D. R. Bes and Z. Szymański, Nucl. Phys. 28 (1963) 42;

Z. Szymaŕski, Nucl. Phys. 28 (1963) 23

5) C. Gustafson, I.-L. Lamm, B. Nilsson and S. G. Nilsson, Ark. Fys. 36 (1966) 613

6) V. M. Strutinsky, Yad. Fiz. 3 (1966) 614 [Sov. J. Nucl. Phys. 3(1966) 449]; Ark. Fys. 36 (1966) 629; Nucl. Phys. A95 (1967) 420

7) V. M. Strutinsky, Nucl. Phys. A122 (1968) 1

8) M. Brack, J. Damgaard, A. S. Jensen, H. C. Pauli, V. M. Strutinsky and C. Y. Wong, Rev, Mod. Phys. 44(1972) 320

9) M. Bolsterli, E. O. Fiset, J. R. Nix and J. L. Norton, Phys. Rev. C5 (1972) 1050

10) D. Scharnweber, W. Greiner and U. Mosel, Nucl. Phys. A164 (1971) 257;

U. Mosel and H. W. Schmitt, Phys. Rev. C4 (1971) 2185

11) B. L. Andersen, F. Dickmann and K. Dietrich, Nucl. Phys. A159 (1970) 337

12) V. V. Pashkevich, Nucl. Phys. A133 (1969) 400 ; A169 (1971) 275

13) S. G. Nilsson, C. F. Tsang, A. Sobiczewski, Z. Szymański, S. Wycech, C. Gustafson, I.-L. Lamm, P. Möller and B. Nilsson, Nucl. Phys. A131 (1969) 1

14) P. Möller, S. G. Nilsson, A. Sobiczewski, Z. Szymański and S. Wycech, Phys. Lett. 30B (1969) 223

15) S. E. Larsson, I. Ragnarsson and S. G. Nilsson, Phys. Lett. 38B (1972) 269

16) G. Andersson, S. E. Larsson, G. Leander, P. Möller, S. G. Nilsson, I. Ragnarsson, S. Åberg, R. Bengtsson, J. Dudek, B. Nerlo-Pomorska, K. Pomorski and Z. Szymański, Nucl. Phys. A268 (1976) 205

17) G. G. Bunatian, V. M. Kolomietz and V. M. Strutinsky, Nucl. Phys. A188 (1972) 225;

A. S. Tyapin, Yad. Fiz. 19 (1974) 263 [Sov. J. Nucl. Phys. 19 (1974) 129]

18) W. J. Bassichis, A. K. Kerman, C. F. Tsang, D. R. Tuerpe and L. Wilets, in Magic without magic; John Archibald Wheeler, a collection of essays in honor of his 60 th birthday, ed. J. R. Klauder (Freeman, San Francisco, 1972) p. 15

19) M. Brack and P. Quentin, in Physics and chemistry of fission 1973 (IAEA, Vienna, 1974) Vol. I, p. 231

20) M. Brack and P. Quentin, in Nuclear self-consistent fields, ed. G. Ripka and M. Porneuf (NorthHolland, Amsterdam, 1975) p. 353

21) M. Brack and P. Quentin, Phys. Lett. 56B (1975) 421

22) S. T. Belyaev, Mat. Fys. Medd. Dan. Vid. Selsk. 31 (1959) no. 11

23) S. G. Nilsson and O. Prior, Mat. Fys. Medd. Dan. Vid. Selsk. 32 (1961) no. 16

24) N. N. Bogoliubov, Dokl. Akad. Nauk SSSK 119 (1958) 244;

N. N. Bogoliubov and V. G. Soloviev, Dokl. Akad. Nauk. SSSR 124 (1959) 1011

25) V. M. Kolomietz, Yad. Fiz. 18 (1973) 288 [Sov. J. Nucl. Phys. 18 (1974) 147]

26) A. S. Jensen and J. Damgaard, Nucl. Phys. A203 (1973) 578

27) L. G. Moretto, in Physics and chemistry of fission 1973 (IAEA, Vienna, 1974) Vol. I, p. 329, and references therein

28) T. Dфssing and A. S. Jensen, Nucl. Phys. A222 (1974) 493

29) M. Prakash, V. S. Ramamurthy and S. S. Kapoor, in Physics and chemistry of fission 1979 (IAEA, Vienna 1980) Vol. II, p. 353

30) G. G. Bunatian, Yad. Fiz. 29 (1979) 10 [Sov. J. Nucl. Phys. 29 (1979) 4]

31) M. Brack and P. Quentin, Phys. Scripta A10 (1974) 163; Phys. Lett. 52B (1974) 159

32) M. Brack and P. Quentin, in Nuclear self-consistent fields, ed. G. Ripka and M. Porneuf (NorthHolland, Amsterdam, 1975) p. 399

33) U. Mosel, P.-G. Zint and K. H. Passler, Nucl. Phys. A236 (1974) 252;

G. Sauer, H. Chandra and U. Mosel, Nucl. Phys. A264 (1976) 221

34) V. S. Ramamurthy, S. S. Kapoor and S. K. Kataria, Phys. Rev. Lett. 25 (1970) 386

35) L. G. Moretto, Phys. Lett. 38B (1972) 393

36) R. Bengtsson, Nucl. Phys. A198 (1972) 591

37) R. K. Bhaduri and S. Das Gupta, Phys. Lett. 47B (1973) 129

38) A. S. Tyapin, Yad. Fiz. 11 (1970) 89 [Sov. J. Nucl. Phys. 11 (1970) 53]; Yad. Fiz. 14 (1971) 88 [Sov. J. Nucl. Phys. 14 (1972) 50]

39) R. K. Bhaduri and C. K. Ross, Phys. Rev. Lett. 27 (1971) 606

40) B. K. Jennings, Nucl. Phys. A207 (1973) 538; see also Ph.D. Thesis, McMaster Univ. (1976) unpublished 
41) B. K. Jennings, R. K. Bhaduri and M. Brack, Nucl. Phys. A253 (1975) 29

42) R. Balian and C. Bloch, Ann. of Phys. 69 (1972) 76, and references therein

43) D. H. E. Gross, Phys. Lett. 42B (1972) 41

44) P. Quentin and H. Flocard, Ann. Rev. Nucl. Part. Sci. 28 (1978) 523

45) C. Bloch and A. Messiah, Nucl. Phys. 39 (1962) 95

46) N. N. Bogoliubov, Nuovo Cim. 7 (1958) 795

47) J. G. Valatin, Nuovo Cim. 7 (1958) 843

48) D. Gogny, in Nuclear self-consistent fields, ed. G. Ripka and M. Porneuf (North-Holland, Amsterdam, 1975) p. 333

49) B. Grammaticos, thèse d'état, Univ. Paris-Sud, Orsay (1977), unpublished

50) J. F. Berger and M. Girod, in Physics and chemistry of fission 1979 (IAEA, Vienna, 1980), Vol. I, p. 265

51) J. Dechargé and D. Gogny, Phys. Rev. C21 (1980) 1568

52) M. Sano and S. Yamasaki, Prog. Theor. Phys. 29 (1963) 397

53) M. Brack and H. C. Pauli, Nucl. Phys. A207 (1973) 401

54) D. Vautherin, Phys. Rev. C7 (1973) 296

55) H. A. Bethe, Ann. Rev. Nucl. Sci. 21 (1971) 93

56) K. Dietrich, in The structure of nuclei (IAEA, Vienna, 1972) p. 373

57) V. M. Strutinsky, Nucl. Phys. A218 (1974) 169; A254 (1975) 197

58) H. Flocard, P. Quentin, D. Vautherin, M. Vénéroni and A. Kerman, Nucl. Phys. A231 (1974) 176

59) M. Brack, in Physics and chemistry of fission 1979, Jülich (IAEA, Vienna, 1980) Vol. I, p. 227

60) T. Johansson, S. G. Nilsson and Z. Szymanski, Ann. de Phys. 5 (1970) 377

61) J. R. Nix, Ann. Rev. Nucl. Sci. 22 (1972) 65

62) H. C. Pauli, Phys. Reports C7 (1973) 35

63) W. H. Bassichis and L. Wilets, Phys. Rev. Lett. 22 (1969) 799;

L. Wilets, in Physics and chemistry of fission 1969 (IAEA, Vienna, 1969) p. 179

64) W. H. Bassichis and D. R. Tuerpe, Phys. Rev. C8 (1973) 541

65) W. H. Bassichis, D. R. Tuerpe, C. F. Tsang and L. Wilets, Phys. Rev. Lett. 30 (1973) 294

66) M. Beiner, H. Flocard, Nguyen Van Giai and P. Quentin, Nucl. Phys. A238 (1975) 29

67) D. Vautherin and D. M. Brink, Phys. Rev. C5 (1972) 626

68) J. W. Negele and D. Vautherin, Phys. Rev. C5 (1972) 1472

69) Y. H. Chu, Ph.D. Thesis, Stony Brook (1977), unpublished

Y. H. Chu, B. K. Jennings and M. Brack, Phys. Lett. 68B (1977) 407

70) H. Flocard, P. Quentin, A. K. Kerman and D. Vautherin, Nucl. Phys. A203 (1973) 433

71) M. Brack, P. Quentin and D. Vautherin, in Int. Symp. on superheavy elements, ed. M. A. K. Lodhi (Pergamon, New York, 1978) p. 309

72) V. M. Strutinsky, in Physics and chemistry of fission 1979, Jülich (IAEA, Vienna, 1980) Vol. I, p. 475

73) E. Werner, K. Dietrich, P. Möller and J. R. Nix, in Physics and chemistry of fission 1979, Jülich (IAEA, Vienna, 1980) Vol. I, p. 501

74) N. Marty et al., Proc. Int. Symp. on highly excited states in nuclei, Jülich (1975) p. 17

75) J. P. Blaizot, D. Gogny and B. Grammaticos, Nucl. Phys. A265 (1976) 315

76) O. Bohigas, A. M. Lane and J. Martorell, Phys. Reports 51 (1979) 267

77) H. Krivine, J. Treiner and O. Bohigas, Nucl. Phys. A336 (1980) 155

78) C. Guet, R. Bengtsson and M. Brack, in Physics and chemistry of fission 1979, Jülich (IAEA, Vienna, 1980) Vol. II, p. 411

79) J. Libert, M. Meyer and P. Quentin, Phys. Lett., 95B (1980) 175

80) S. A. Moszkowski, Phys. Rev. C2 (1970) 402;

S. Köhler, Nucl. Phys. A258 (1976) 301;

B. Rouben, J. M. Pearson and G. Saunier, Phys. Lett. B42 (1972) 385

81) D. Kolb, R. Y. Cusson and H. W. Schmitt, Phys. Rev. C10 (1974) 1529

82) E. Wigner, Phys. Rev. 40 (1932) 749;

J. G. Kirkwood, Phys. Rev. 44 (1933) 31

83) A. Voros, thèse d'état, Univ. Paris-Sud, Orsay (1977), unpublished

84) B. K. Jennings, R. K. Bhaduri and M. Brack, Phys. Rev. Lett. 34 (1975) 228

85) M. Brack, Habilitationsschrift, ILL Preprint 77 BR 346 S (Grenoble 1977)

86) W. D. Myers and W. J. Swiatecki, Nucl. Phys. 81 (1966) 1 
87) W. D. Myers, Nucl. Phys. A145 (1969) 387;

W. D. Myers and W. J. Swiatecki, Ann. of Phys. 55 (1969) 395

88) V. M. Strutinsky and F. A. Ivanjuk, Nucl. Phys. A255 (1975) 405;

F. A. Ivanjuk and V. M. Strutinsky, Z. Phys. A286 (1978) 291 ; A290 (1979) 107

89) C. F. v. Weizsäcker, Z. Phys. 96 (1935) 431;

H. A. Bethe and F. Bacher, Rev. Mod. Phys. 8 (1936) 82

90) R. A. Berg and L. Wilets, Phys. Rev. 101 (1956) 201;

L. Wilets, Rev. Mod. Phys. 30 (1958) 542

91) T. H. R. Skyrme, Phil. Mag. 1 (1956) 1043; Nucl. Phys. 9 (1959) 615;

K. Kumar and R. K. Bhaduri, Phys. Rev. 122 (1961) 1926

92) H. A. Bethe, Phys. Rev. 167 (1968) 879;

K. A. Brückner, J. R. Buchler, S. Jorna and R. L. Lombard, Phys. Rev. 171 (1963) 1188

93) R. J. Lombard, Ann. of Phys. 77 (1973) 380

94) J. P. Hohenberg and W. Kohn, Phys. Rev. 136 (1964) B864

95) N. D. Mermin, Phys. Rev. 137 (1965) Al441

96) M. Brack, B. K. Jennings and Y. H. Chu, Phys. Lett. 65B (1976) 1

97) B. Grammaticos and A. Voros, Ann. of Phys. 123 (1979) 359; part II, to be published

98) D. A. Kirzhnits, JETP (Sov. Phys.) 5 (1957) 64;

C. H. Hodges, Can. J. Phys. 51 (1973) 1428

99) O. Bohigas, X. Campi, H. Krivine and J. Treiner, Phys. Lett. 64B (1976) 381

100) C. Guet and M. Brack, Z. Phys., A297 (1980) 247

101) C. Guet, H.-B. Håkansson and $M$. Brack, to be published

102) M. Brack, B. K. Jennings and $P$. Quentin, to be published

103) R. Bengtsson and P. Schuck, Phys. Lett. 49B (1980) 321

104) R. K. Bhaduri, Phys. Rev. Lett. 39 (1977) 329

105) M. Durand, M. Brack and P. Schuck, Z. Phys. A286 (1978) 381

106) M. Durand, P. Schuck and M. Brack, Z. Phys. A296 (1980) 87

107) J. Bartel, R. K. Bhaduri, M. Brack, M. Durand and P. Schuck, to be published

108) M. Brack, Phys. Lett. 81B (1977) 239

109) C. M. Ko, H. C. Pauli, M. Brack and G. E. Brown, Phys. Lett. 45B (1973) 433; Nucl. Phys. A236 (1974) 269

110) J. Meyer, P. Quentin, H. Flocard, H.-B. Håkansson and M. Brack, to be published

111) C. Guet, H.-B. Hăkansson and M. Brack, Phys. Lett. 97B (1980) 7 